University of Redlands

\title{
Automating Quality Control in a Crowd-Sourced Marine Mammal Observation Database
}

A Major Individual Project submitted in partial satisfaction of the requirements

for the degree of Master of Science in Geographic Information Systems

by

Rachel Guttmacher

Douglas Flewelling, Ph.D., Committee Chair

Mark Kumler, Ph.D.

July 2015 
Automating Quality Control in a Crowd-Sourced Marine Mammal Observation Database

Copyright $(C) 2016$

by

Rachel Guttmacher 
The report of Rachel Guttmacher is approved.

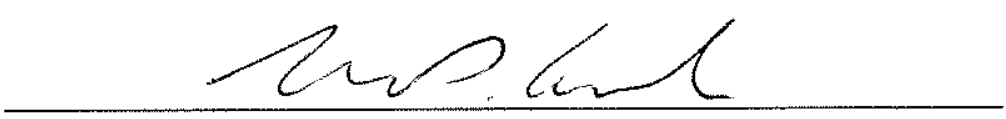

Mark Kumler, Ph.D.

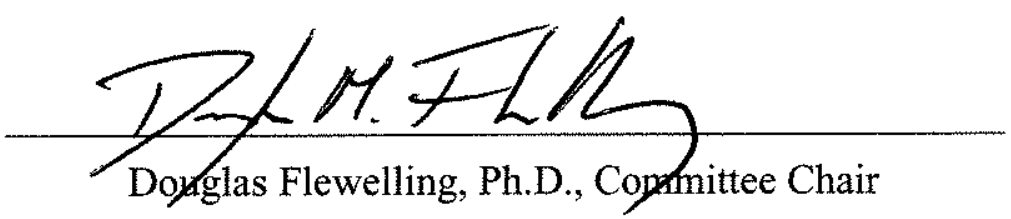

October 2016 



\section{Acknowledgements}

Thank you to my advisor, Dr. Douglas Flewelling, for all of his guidance and assistance for this project from proposal through to completion. I am also extremely grateful to Dr. Lei Lani Stelle, who approached the Masters in GIS Program students with the request for assistance that led to this project. I would also like to thank Melodi King and Matthew Flewelling for assistance in brainstorming and troubleshooting and remaining on call for help throughout the year.

I would also like to thank Cohort 26 for the support given from Day 1 to graduation. Through all the presentations, feedback requests, practice runs, and opinions provided, this project would not have been as successful or as great an experience without all of you here with me.

To Andrea Barrios, the Program Coordinator, thank you for helping with all the scheduling, the help to keep on track, the "You can do it!" conversations exchanged in the office, and all the work you have put in to the MS GIS Program this year.

A big thanks finally to my family and friends outside of Redlands. Regardless of how far away, you all played a part in me getting through this project. There were plenty of ups and downs this year, but it is thanks to your support that I made it.

Thank you all! 



\begin{abstract}
Automating Quality Control in a Crowd-Sourced Marine Mammal Observation Database

by

Rachel Guttmacher

An Android mobile application, the Whale mAPP, utilizes crowd sourcing data collection to gather data about marine mammals. The use of volunteered geographic information invites data inconsistencies including clusters of similar data submissions, incorrect species identification, and on-land observations caused by signal drop or user error. Prior to this project, these issues went unnoticed in the resulting dataset, leading to significant reliability problems. This project uses Python code to automate the flagging of these problematic points. The project also simplifies the process of accepting new contributors. These Python scripts ensure data quality issues are evident in resulting crowd-sourced datasets prior to use by marine biologists and other interested parties.
\end{abstract}





\section{Table of Contents}

Chapter 1 - Introduction ................................................................................................ 1

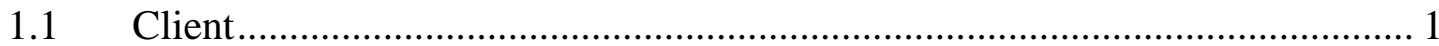

$1.2 \quad$ Problem Statement ........................................................................... 2

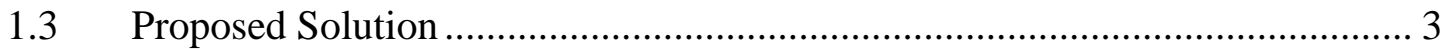

1.3.1 Goals and Objectives ………………………......................................... 3

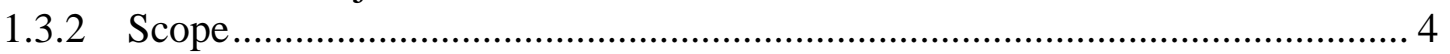

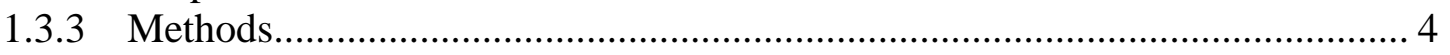

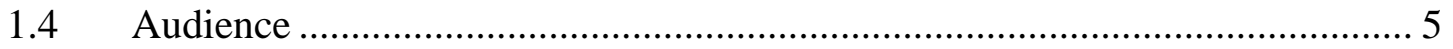

1.5 Overview of the Rest of this Report ......................................................... 5

Chapter 2 - Background and Literature Review ............................................................. 7

$2.1 \quad$ Volunteered Geographic Information ....................................................... 7

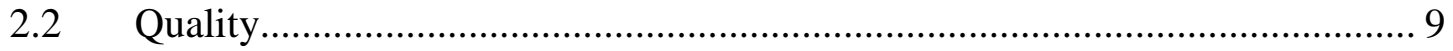

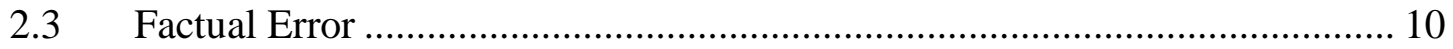

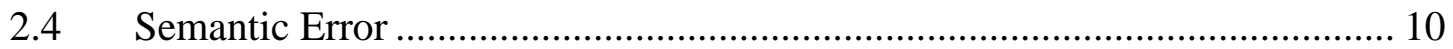

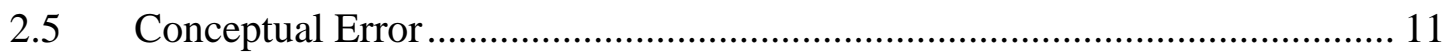

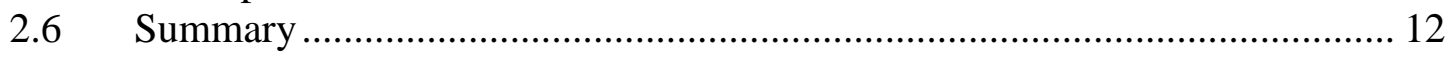

Chapter 3 - Systems Analysis and Design........................................................................ 13

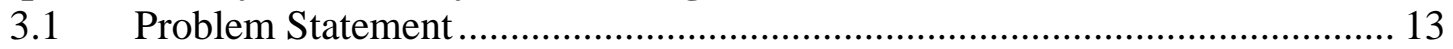

3.2 Requirements Analysis ……………………….................................... 13

3.3 System Design ................................................................................... 14

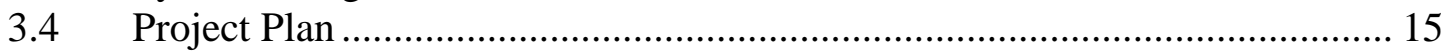

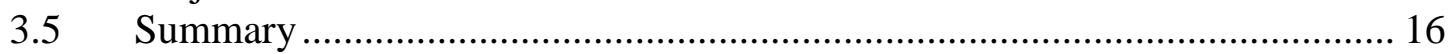

Chapter 4 - Database Design.................................................................................................. 17

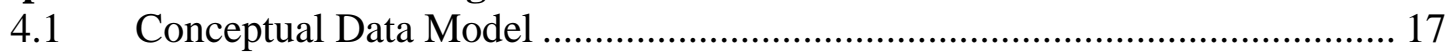

4.2 Logical Data Model ………………………...................................... 18

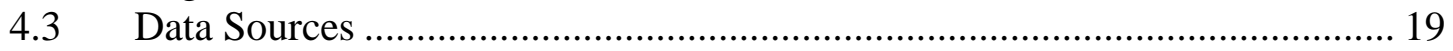

4.4 Data Collection Methods ...................................................................... 20

4.5 Data Scrubbing and Loading …………………...................................... 21

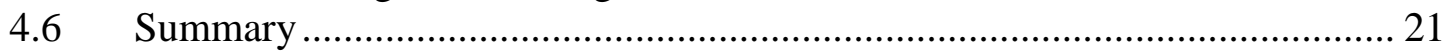

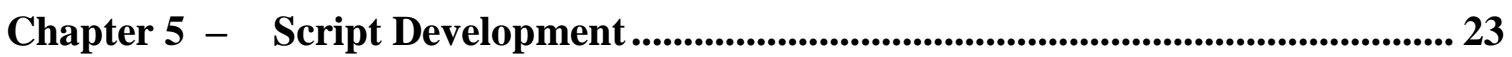

$5.1 \quad$ Quality Check Scripts …………………………................................... 23

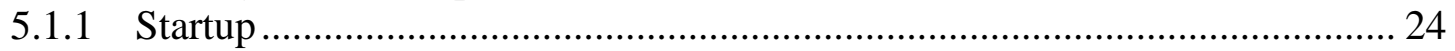

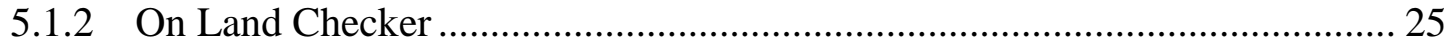

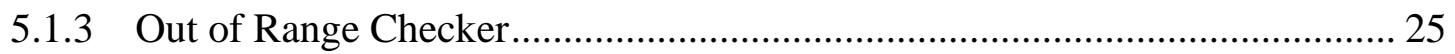

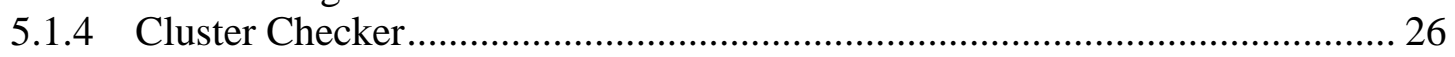

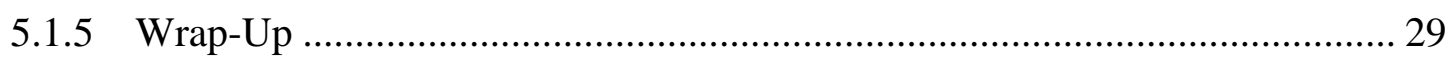

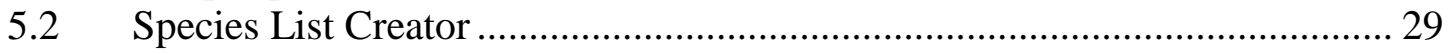

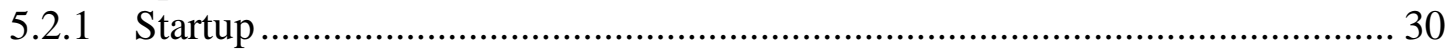

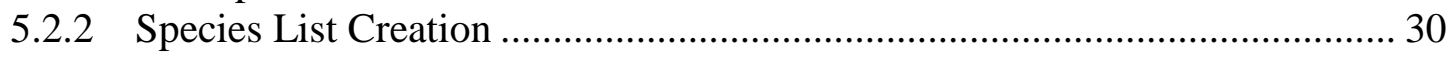

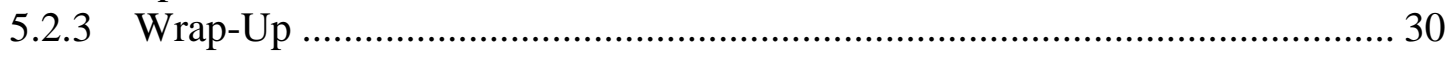

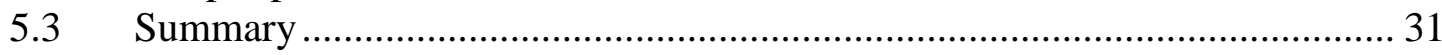


Chapter 6 - Results and Analysis........................................................................ 33

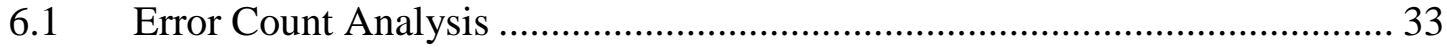

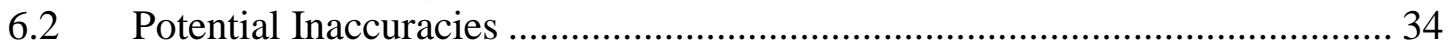

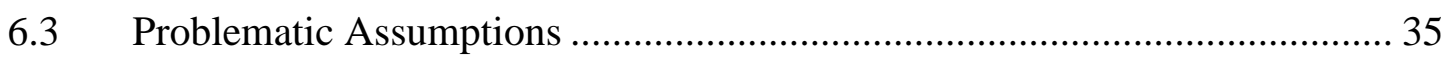

Chapter 7 - Conclusions and Future Work ................................................................. 37

Works Cited 41 


\section{Table of Figures}

Figure 1-1 The Bottlenose and the Franciscana Dolphin ..................................... 2

Figure 2-1 OpenStreetMap.Org, 2016..................................... 8

Figure 2-2 WikiMapia.Org, 2016......................................... 8

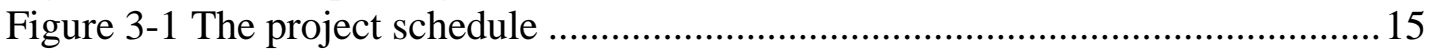

Figure 4-1 The conceptual data model: prior work and new work ......................... 18

Figure 4-2 The logical data model ................................................................. 19

Figure 4-3 The California Sea Lion: source versus feature created ..........................23

Figure 5-2 Summary of the quality checker script............................................. 23

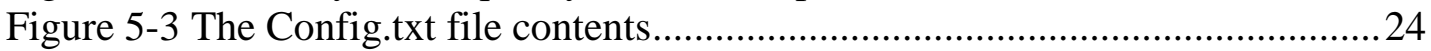

Figure 5-4 The On Land Checker workflow.........................................................25

Figure 5-5 Out of Range Checker workflow .......................................................26

Figure 5-6 Cluster Checker workflow ................................................................28

Figure 5-7 Summary of the species list creator script...........................................29

Figure 7-1 Hypothetical Concave Hull Analysis ................................................. 39 



\section{List of Tables}

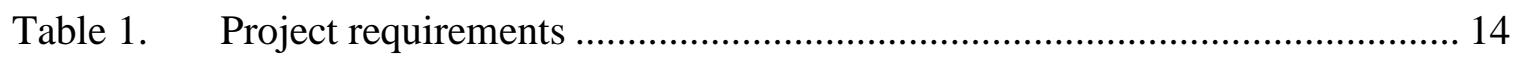

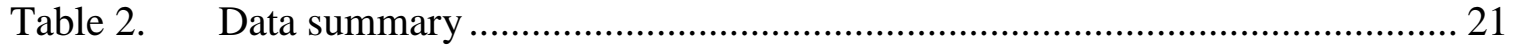

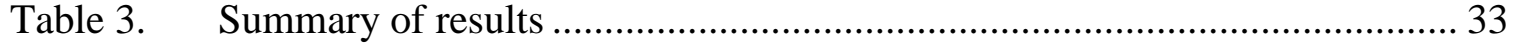





\section{List of Acronyms and Definitions}

IDE Integrated development environment

MMOP Marine mammal observation point

VGI Volunteered geographic information 



\section{Chapter 1 - Introduction}

Oceans cover just under three quarters of the Earth's surface and play a major role in the ebb and flow of life. Marine mammals often serve as reliable indicators of the state of the oceans. Collecting observational data on marine mammals can be both tedious and expensive. Recently a mobile application was developed that attempts to utilize volunteers on marine outings. Use of the public offers a large volume of data in a shorter period than sending a marine biologist into the field. Melodi King, a previous student at the MSGIS program, produced the Whale mAPP, an application usable on Android mobile phones to record boat paths while also providing a quick form for recording marine mammal observations. This application allows the public to assist in data collection while they record their own trips for personal use through an online application.

This process has extensive error possibilities most often due to limited user knowledge of marine mammals, redundant observations, and problematic mobile network connectivity. Until now, there was no way to assess the type and extent of these errors. In order to fix the quality of the data every point in the dataset needed to be examined to identify and resolve problems. This process was tedious and took time and attention to detail. In order to resolve these issues a set of automatic tools was created that identify these errors using spatial characteristics such as containment analysis, distance between points, and other ArcGIS geoprocessing tools in the form of Python scripts.

The Whale mAPP populates a drop-down list of species to identify using information collected from users requesting access to the Whale mAPP. Previously these lists had to be produced manually by requesting species list information from the new user. Because the application eventually will become public, this populate-area-by-request process would have been unable to handle the number of requests caused by the worldwide release of the application. The final role of this project was to resolve this problem by using marine mammal range shapefiles to populate these lists instead of leaving the user to request a list of species to include.

\subsection{Client}

Dr. Lei Lani Stelle is a marine biologist currently employed by the University of Redlands. She has lectured on topics ranging from river otters to whales to sea lions. Dr. Stelle approached the MSGIS Program at the University of Redlands two years ago to propose a project for a prior MSGIS student. Together, Dr. Stelle and Ms. King produced significant work with the start of the Whale mAPP. Throughout this project, Dr. Stelle was available for frequent discussions in regards to further application development and provided data for testing the error querying process. She also was available to give feedback as the project evolved. Ms. King was also available as a resource and remained on-call for further questions as to the original development of the application and suggestions for future corrections and update processes. 


\subsection{Problem Statement}

Dr. Stelle requested further assistance in order to automate the identification of data errors to enhance the reliability of data collected through the Whale mAPP application. The Whale mAPP operates on the assumption that volunteers have the knowledge to make proper observations. Identification of various species can be difficult for inexperienced observers. For example, two similar-looking species of dolphins, the Bottlenose and the Franciscana dolphins, inhabit the waters off Uruguay, Argentina, and Brazil. The Bottlenose may be found off California; the Franciscana never migrates this far. A Brazilian, vacationing in California could readily mischaracterize a Bottlenose as a Franciscana (Figure 1.1). A post-collection tool made in this project flags data that geographically do not match species distribution to assist with data accuracy awareness.

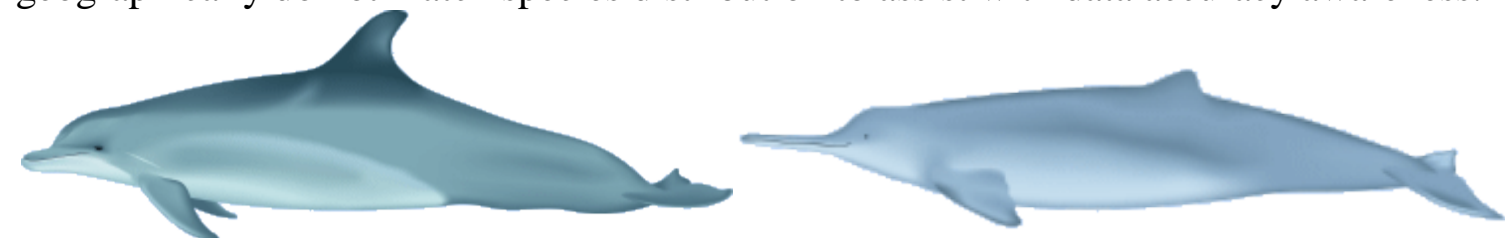

Figure 1-1 The Bottlenose and the Franciscana Dolphin (Comparison Not to Scale)

A secondary problem often occurs if more than one person using the Whale mAPP is on a boat at the same time. If a vessel with two recording observers spots a humpback whale and both record the sighting on the Whale mAPP, the dataset resulting from that day's trip would include two humpback whale sightings. If these data are mistaken as individual sighting data, this redundancy could be analyzed as a pod of whales rather than redundant observations. A tool created in this project will flag these redundant sightings as clusters using variables such as distance between points (up to a certain threshold), the time and date of the observation, and the species observed. Should these three factors combine within certain limits, the tool flags the observations for review.

Because the Whale mAPP relies on a device's ability to fetch location information, when signal fails, points may be assigned not to the location they were made, but rather to the location at which a device reconnects to a network. This can be caused by roaming restrictions or complete lack of signal, as often occurs offshore. These points then appear in the database to represent a marine mammal located on land. Users may also attempt to test the application while on land. To resolve the issue of on-land observation errors, spatial queries were produced to flag land-based marine mammal observation points (MMOPs) so they can be disregarded or fixed.

A final tool created during this project assists in producing species lists for new user locations. This tool takes an input location for a new user and searches for all species within a buffer distance specified by the user, and outputs a list of all species expected in that area. This tool allows for quicker introduction of new users to the Whale mAPP. The Whale mAPP can then be programmed to populate the drop down list of species using the output from this tool. 


\subsection{Proposed Solution}

In order to resolve the above problems, a set of ArcGIS Python Scripts was created to automate quality control post-collection. These scripts allow for the processing of data while ensuring a technique for flagging future data before analysis is done on the resulting database. These tools are best handled by running all scripts created by this project at certain time intervals depending on the rate of data collection. This rate will be set by the user as needed. The resulting database shows data that have been flagged for review for incorrect ID, redundancy, or on-land errors. The user then chooses to discard or edit these points at his or her discretion. The use of the marine mammal range shapefiles to populate the drop down list in the Whale mAPP will be incorporated into the application by a programmer after this project. The approach of using ArcGIS Python Scripts allows the functionality of ArcGIS Geoprocessing tools to be applied to multiple files at once, creating a consistency of data quality.

\subsubsection{Goals and Objectives}

The deliverables of this project include a set of tools that increase the quality and usability of data collected by the Whale mAPP. There were three main goals of this project.

The first goal of the project was to create habitat shapefiles. Early in the project, reliable distribution maps of marine mammals throughout the world were identified through a discussion with the client. Many textbooks, guides, and websites include small range maps that display the general area in which a marine mammal can be found. These maps were digitized by hand into shapefiles used for the range check tool and population of species lists.

The second goal was to create ArcGIS Python scripts that test data quality. This set of tools flagged data that are likely to have been made in error, from either duplicate sighting errors, attribute errors, or location errors. The scripts use ArcGIS Geoprocessing tools to flag points for review. Data that have been flagged can then be more efficiently reviewed by the end user. The dataset is kept in the database and corrected or removed as determined by the data reviewer. There were three scripts created to achieve this goal.

The first script was the duplicate sightings script. This tool uses factors such as the species identified, time and date of observation, and the distance between observations to determine if they are duplicates. If they meet the criteria set by this script, the points are flagged for review to avoid duplicate observation points. Attributes are manually reviewed between the two observations to identify whether they are duplicative reports based on differences such as the number of animals spotted in a pod, their behavior, and photographs submitted with the observation, and are corrected at the discretion of the data reviewer.

The second script was the out-of-range script. This tool uses the shapefiles created in the first goal to compare collected observations with the ranges of the species identified. The points are flagged for review as a potential misidentified species if they do not fall within the border of the range shapefiles. This helps to filter out user error. Photos taken during the user's trip through the application are available for review by those checking these points. Should a photo allow species identification then these points may be kept or discarded per the data reviewer's decision. 
The final quality control script was the on-land script. This tool assists in flagging points that received incorrect location data due to network errors. If points receive location data once on-land instead of at sea, this tool identifies these land-based observations and flags them for review. Unlike the other types of errors, the data resulting from this error may be fixable if the boat trip path stayed intact and if the observation has a time stamp. This can be used as a proof of concept to encourage time stamps to be implemented in the Whale mAPP to allow adjusting of the incorrect data into useable points. The decision to correct points would ultimately be made by the individual reviewing flagged data.

The final goal of the project was to create the species list script. This script uses the ranges created at the beginning of the project to generate species lists for new users. This script helps by standardizing the species lists for all users. It also speeds up the process of programming new users into the application by automating part of new programming that would need to be entered into the Whale mAPP.

\subsubsection{Scope}

Currently in beta testing, the Whale mAPP is only available to a limited number of locations including the Caribbean region, the coast of California, and near Vancouver Island. The application is intended for worldwide use, and the tools and range data created in this project is worldwide, allowing for continued use of these scripts once the application becomes publicly available.

Data digitizing and script programming consumed the largest portion of the time for this project. Digitizing and script programming encompassed about $40 \%$ of the project duration. The remaining $20 \%$ included research, testing, client meetings, and project write-up.

The major technical components of this project included Python scripting and ArcGIS Geoprocessing tools. Digitizing work was done within ArcGIS and programming was done within PyScripter. Work was completed on the laptop provided by the University of Redlands and the dataset was copied from the Whale mAPP dataset by way of a zipped file. There was no need for extra hardware or software. When a test dataset was needed, a point file was made manually by adjusting sample Whale mAPP data points to display errors to test. Aside from the cost of transportation to Dana Point harbor to test the Whale mAPP, which was paid for out of pocket, there was no cost to this project.

\subsubsection{Methods}

There were eight major steps in this project. First, marine mammal range information was collected from reliable sources. Concise sources for ranges of marine mammals were readily available. Determining the reliability of various sources, however, did pose a challenge. Sources were checked with the client in order to get feedback on the accuracy of the ranges included in each source.

The second step was to collect test data in order to start building scripts. While this step could have gone after the third step listed below, it was best to collect test data early on in order to avoid any risk of loss of data on which to test quality assurance tools. Obtaining Whale mAPP data before any significant work was done was prudent in case 
of system failure and consequent data losses. Sample data was provided directly from the current Whale mAPP dataset.

The third step was included the creation of range shapefiles from the information gathered in step one. This took a significant amount of time. Several attempts were made to reach contacts for premade range shapefiles displayed on websites, but contact was not returned. Instead, range shapefiles were produced manually by trimming an ocean shapefile to fit the ranges specified by the sources approved by the client.

The fourth step was to review all range shapefiles with the client. This step provided an opportunity to receive feedback and request changes to the project. Once confirmation was given that the shapefiles would work for our needs, the project continued.

The fifth step began the programming. The quality check scripts and the species list scripts were created and debugged in this step. Once this step was done, the majority of the work on this project was complete.

The sixth step included script testing. Whale mAPP data collected in step two was used in this step to test the script and current data quality. Once the tools flagged the proper data, the project continued.

The seventh step included another review. The scripts were tested for efficiency in this step as well.

The final step was the completion of the project. This included the write-up, submission to the University of Redlands and to the client, and the closure of the project. This step also included a presentation at the Esri User Conference in San Diego, California.

\subsection{Audience}

The audience for this project includes individuals with limited GIS experience. The client has general knowledge of GIS themes and theories, but has no formal training on how to use software such as ArcGIS. The client has expressed a desire to learn, but for the purposes of this project, the tools are created to be user friendly for individuals without GIS software knowledge.

The report contained herein is written towards individuals who have knowledge of general GIS themes and limited knowledge of ArcGIS software. This report becomes more technical when discussing the files and programming necessary to create these quality control tools, but for the most part should be understood by basic GIS users.

\subsection{Overview of the Rest of this Report}

The report following this chapter contains information as to the background, production, and completion of this project. Chapter 2 summarizes background research done before the start of this project. Chapter 3 discusses the planning phase of the project including requirements, system design, and the project plan submitted to the MS GIS Program. Chapter 4 handles data information such as data models, sources, and quality checking to insure the highest quality data possible for this project. Chapter 5 covers project implementation and deployment, and contains the most information on the production portion of the project. Chapter 6 contains the results and analysis of the project, including discussions of problems that occurred along the way. Finally, Chapter 7 includes the 
project conclusions and a discussion of potential future work on the subject. This report will close with works cited. 


\section{Chapter 2 - Background and Literature Review}

Because volunteered geographic information (VGI) may be collected by untrained public users, the quality of resulting data may vary greatly. In order to have a better understanding of this data, checks and balances can be made to filter through these potential errors and get a better understanding of the dataset. Research was completed on past work to search for ideas and ways to approach this project. After examining studies about VGI, quality assurance, and quality control, a plan was built to resolve the issues the Whale mAPP system is currently facing. The three issues cropping up - including incorrect species identification, land-locked marine mammal sighting reports, and duplicate observations from multiple users on the same boat - were categorized into three items of concern: factual, semantic, and conceptual errors. Prior research also covered how to increase the quality of VGI and how to respond to connectivity issues. Examining these previous studies for ideas with the Whale mAPP was beneficial even when the studies examined were not marine-based. The frameworks and basic ideas of many studies are interchangeable, and spatial analysis and programming can produce useable results in any project.

\subsection{Volunteered Geographic Information}

The phrase "volunteered geographic information" was first coined in a paper titled "Citizens as Sensors: the World of Volunteered Geography" (Goodchild, 2007). Goodchild characterized VGI as a sub-section of user-generated content. Though Goodchild coined the phrase, the concept of the public contributing to studies has been spoken of by many researchers, and has been pointed to under several alternative terms such as crowdsourcing, public participation GIS, citizen science, and even participatory GIS. In this day and age many companies have realized the value behind using public input and have welcomed information from many users. On the internet, projects such as OpenStreetMap, first available in 2004, and Wikimapia, released in 2012, are well known. Both seek information from the public in labeling and identifying the world. 


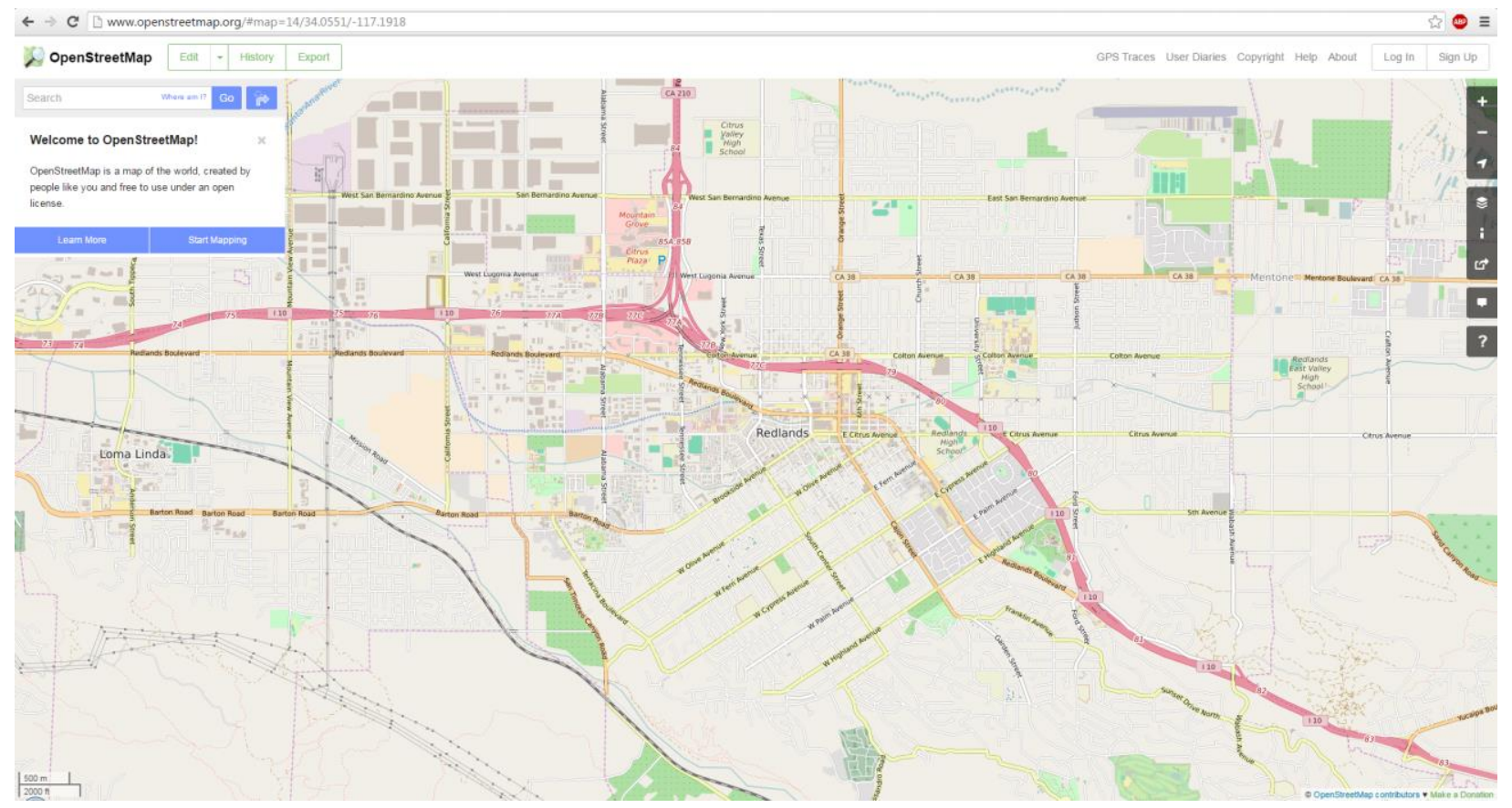

Figure 1-1 OpenStreetMap.Org, 2016

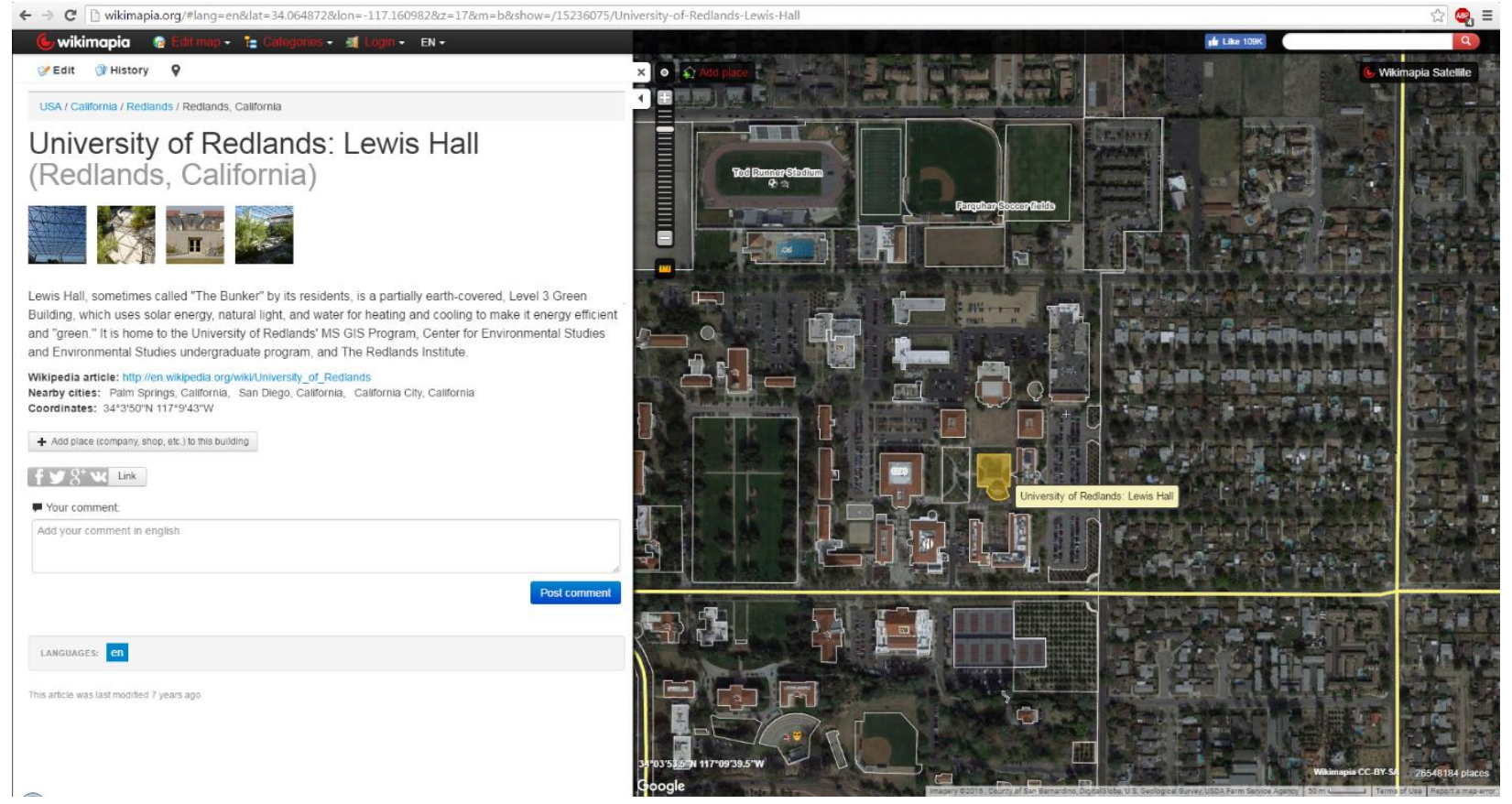

Figure 2-2 WikiMapia.Org, 2016

Goodchild also cautioned about the primary concern for all VGI projects: reliability. In traditional data collection run by large companies a substantial amount of time and money is put forth to ensure that the individuals collecting data and the equipment used are of the highest quality. This often allows these larger companies to become known as authoritative data sources - sources which can be relied upon for correctness, may have been created for a specific use by paid professionals, and may be available by only 
limited access (Coleman, 2013). VGI, however, is asserted data in that it does not follow standards or quality checks prior or during acquisition, and many of the collectors are untrained, less-detailed, and sometimes careless. While involving the public increases the volume of data received, the quality of the data may in fact drop overall.

The Whale mAPP was designed to collect a large volume of data from the public, but because the incoming data had not yet been reviewed for inconsistencies, the need for this project arose. This project seeks to resolve this issue by implementing quality checks and standards into post-collection evaluation. While this does not increase the quality of the incoming data itself, it allows for a buffer between data collection and data users, during which an analysis of the data consistency can be completed.

\subsection{Quality}

Many individuals and groups have attempted to define quality. These attempts extend well beyond the boundaries of geographic information systems studies. When comparing various resources, some consistent themes are repeated (Devillers \& Jeansoulin, 2006; Kerski \& Clark, 2012; United Nations, 1998) such as:

1. Lineage: what is the source of the data?

2. Positional accuracy: how close data are to true location?

3. Attribute accuracy: how close to true value are the attributes?

4. Logical consistency: can data interrelationships be maintained without juxtapositions?

5. Completeness: does the data capture all features needed?

All five of these items are of great importance when examining the quality of a dataset. In this project, the three we are primarily focusing on are positional accuracy, attribute accuracy, and completeness or, in this case, over-abundance. If our position collection is incorrect, we will get erroneous data in unusual locations. If the attributes are entered incorrectly, data may give the impression of changes in habitat or population ranges. If data is overabundant due to multiple individuals entering the same sighting, then these duplicate sightings may unbalance the dataset. We already know the lineage of the data: the public. While end users need to be careful about trusting this source, the whole reason behind adding these checks to post-processing decreases potential problems that could arise from non-authoritative lineage. Completeness is a bit rhetorical - at this point there is no defined end goal for the Whale mAPP, so the dataset will always be incomplete going forward as the dataset continues to change daily.

Many sources also mention one of the most important aspects of quality: the fitness of use. A dataset may be of high accuracy in all of the aforementioned categories, but if the dataset is not related to an end user's project, the dataset will be unneeded. Several of the sources break fitness of use down even further, identifying that the quality and use of a dataset differs from individual to corporate needs and interpretations of the data (Coleman, 2013), from producer's perception to user's perception (Stein, Shi, \& Bijker, 2009) but also from organization to organization (Perry, 1991). The different variables

and requirements for using datasets change the interpretations of quality. In order to make 
datasets the most widely usable, data producers must always work with the end users in mind.

The Whale mAPP data was designed with flexibility in mind for the outputs. While the application does guide the user with pre-populated fields and suggestions, the end data users can use the data how they see fit, as long as they are aware of the potential errors and limitations of the dataset during production and post-production quality checks.

\subsection{Factual Error}

Factual error can occur in many ways though it is most often caused, as in this project, by a lack of knowledge or training of the individuals recording data. Marine mammals can be notoriously difficult to identify as many species look and behave similar and they spend the majority of their time underwater and out of sight. Therefore, it would be safe to assume that some of the incoming data may be misidentified species. While training would be the ideal way to resolve this issue, training every individual prior to use of the Whale mAPP is neither feasible nor realistic. Post-collection processing however can examine this error and start us off in the right direction of understanding the resulting data and their quality. Therefore, in order to research how to handle this issue, studies on factual error were examined. One author mentions several different ways to evaluate factual error (Veregin, 1992). Ground-truthing is most commonly used for geographic data (Steinberg \& Steinberg, 2015). Because many geographical features are static, users can travel to a location to compare results collected from a study with reality. For example, a comparison can be made between an interpolated soil sample and a test conducted onsite at the interpolated location. Comparing and contrasting these results could identify user and producer inaccuracies. If a street is labeled as Fifth Avenue on a map and a user travels to the location marked, the user can check whether the street signs actually state "Fifth Avenue".

While ground-truthing is a viable solution for many projects, the Whale mAPP is intended to record observations that are only a specific instance in time. While certain marine mammals may frequent specific locations, such as a colony of seals sunbathing every morning on a jetty, most marine mammals spend their entire lives traveling back and forth from feeding and breeding grounds, so a whale may be around one section of coastline only for a day or two, or may linger longer depending on the season. Dolphins and porpoises are less likely to travel, but identifying the same individual or even the same pod may prove futile. We are therefore unable to implement ground-truth assessments with Whale mAPP data. Instead, we must do the best with what we have available to us by assessing the likelihood of a point's validity using known frequented locations for each species. If a species is recorded outside of its predominant habitat, this could indicate the likelihood of a factual error. In some cases, some users may also take a photo of the individuals recorded, so examining photography can be used an alternative factual check.

\subsection{Semantic Error}

Semantic error can cover a wide range of issues, from programmatic syntax to technical problems. In this project the semantic error examined is in regards to positional accuracy. 
The Whale mAPP is designed for users to collect data and assign locations per read-outs from the Global Positioning System (GPS) built in to their devices. This works well for devices used in populated areas such as cities and towns. This, however, is less reliable in areas of poor reception, namely mountains, rural locations, and out in the ocean where phone companies are unable to install towers to increase connectivity. With the loss of adequate reception, the degrading of positional accuracy usually follows.

While positional technology is still relatively new compared to the length of time humans have been trying to put themselves on maps, there are still a number of papers that cover the complex issue of resolving connectivity problems post-collection. One study of particular interest examines positional accuracy in vehicles (Carstensen, 1998). When accidents occur on the road, some cars have built-in alert systems that contact emergency dispatch. In such emergencies, the amount of time taken to find the driver is critical and may make a difference in the outcome of life or death. Systems such as Mayday and OnStar were examined in this particular study. A set of filters is used to rule out coordinate points which then narrow down the user's location, increasing efficiency for the emergency crews to locate the vehicle. The study does caution that it is not a foolproof technique. Some legitimate coordinate points are discarded by the filters, resulting in false negatives. Other points may be inaccurate but considered by the filters as false positives. False negatives and false positives are key in determining inconsistencies which can then lead to a higher quality product.

The concept of overlap is also extremely important for this project. Many studies use overlap as an accuracy check (D'Antonio, Fogliaroni, \& Kauppinen, 2014; Koukoletsos, 2012), and such rules are so popular that many different tools for different types of overlaps exist in Esri software alone - Erase, Identity, Intersect, Spatial Join, Symmetrical Difference, Union, Update, Merge, Append, the list goes on. Luckily much of the world works with binary tests. A test either is or is not true. In this case, for most marine mammals, a marine mammal listed as observed on land may have been recorded incorrectly. Using tests such as the overlapping tools mentioned, we can quickly identify which input features overlap the shore. A fourth paper attempts to handle errors in a direction opposite to this project - by flagging points that occurred in water as unlikely locations. The study involves checking the location of land-growing plant samples (Hijmans, Schreuder, De la Cruz, \& Guarino, 1999). When points are located within waterbodies there is cause for concern that the point may not be valid. In such a case, ground-truthing can assist in validating whether a location truly is or is not on land, as the structure of the land changes slowly enough that most project staff can return to a site for a re-check. For example, a dataset may not show that an island sits in the middle of a lake, but visiting the location may reveal the island, thus validating that the point is in fact a valid point. In the case the project for the Whale mAPP data, points that show as on-land may still be in the water. A GIS project can only be so accurate to the location of a shoreline, especially when handling seasonal embankments. Some species can also be found on shore, such as seals and sea lions. The validity of the points would still need to be checked post-quality check, but this would at least remove some of the leg work.

\subsection{Conceptual Error}

One of the most difficult issues about readily available data is the potential for an end user to misunderstand the collection process and interpretation of data collected (Decker, 
2001). One could assume that the primary reason for collecting data with the Whale mAPP is to obtain population and density assessments. The issue with this assumption is that each incoming point stands for one observation. This one observation could correspond to anything from one whale to a thousand dolphins, depending on the recorder's understanding of the collection process or the end user's understanding of the usage. We also run into the issue of two users who may be collaborating together with Whale mAPP and may accidentally submit similar observations about the same breaching humpback whale. The database would then record two separate humpback whale points despite the event only occurring with one whale.

In many cases, the more submissions with similar data, the better the quality of the dataset. Repeated similar observations may in fact support the validity of each other's observations. If five people reported a red convertible driving down a road, it's more likely a red convertible did pass by than if one individual reported such an occurrence by him or herself. Haklay, Basiouka, Antoniou, and Ather (2010) examine the property of many eyes on the same problem, termed Linus' Law, by reviewing studies that compare OpenStreetMap data against premade authoritative datasets. Haklay examines the difference between accuracy in London and accuracy in all of England in an attempt to prove or disprove the application of this law to VGI. The study successfully showed that there was a relationship between the number of users and the quality of the dataset, though the relationship between the two factors was not linear in nature. This seems to indicate that an increase in observations should increase the quality of the dataset.

A study by Vidal-Filho, Lisboa-Filho, de Souza, and dos Santos (2013) looks at MossoróCrimes, a platform for VGI in the city of Mossoro, Brazil. Users can submit incidents and comment on other crimes already in the dataset. The theory behind the commenting is to utilize collaboration to increase the quality of information in the GIS. The users would then be able to self-police the quality of the data, building collaboration and reliability amongst each other as users of the same services. While this was an ideal use of increasing quality by increasing contributors, ultimately the participation in the project was lower than expected and thus went unutilized.

The general consensus of these studies is that redundancy is ideal in many crowdsourced applications. In a perfect world, perhaps the Whale mAPP would eventually gain enough users that they could collaborate and confirm or refute claims by other users in the database. Unfortunately, the dataset as it stands becomes skewed when multiple observations are entered with the same information. Ultimately, there was a need to identify these similar observations for potential redundancy. That is where this project proceeded forward with conceptual error.

\subsection{Summary}

While there were many articles covering volunteered geographic information, quality, and the three types of error confronted in this project, no article was sufficiently on point to feel confident that there was a better solution than the approach taken in this project. The sources discussed above provided for great brainstorming ideas and starting points for conversations with colleagues, and this project will also contribute going forward to help others to study VGI and quality control with a new approach to evaluating data postcollection. 


\section{Chapter 3 - Systems Analysis and Design}

Chapter 3 covers the initial stages of this project. It will first reiterate the problem and then discuss the project requirements. It will also list the design components and describe how the components fit together. The project plan will then be covered, as well as changes that took place between initial planning and the launch of the project.

\subsection{Problem Statement}

Marine mammal observations collected by the crowd-sourced application the Whale mAPP have encountered several data issues. When mobile device reception fails or users want to test the app on land, points appear to indicate that a marine mammal only found at sea has been spotted on land. In addition, a user with limited identification knowledge may misidentify species, causing questionable data submissions. Data will also become more clustered as more users start using the Whale mAPP, causing multiple sightings of the same individual or group to skew the dataset. These data issues need to be flagged for an end user to be better informed of data quality before use. The project described in this report aims to flag these questionable data points through several checks and filters.

\subsection{Requirements Analysis}

When planning for a project it is imperative to understand the end goal. Based on the client's needs, several requirements were compiled and ranked by importance (Table 1).

Critical functional requirements included that the three quality control scripts produced in this project would correctly flag all sightings located on land, all sightings that may be misidentifications as identified as out of range, and all cluster sightings. The fourth script produced had to be capable of receiving an input location from the user and outputting a species list as identified by the range shapefiles.

Other requirements included that the tool should alert the user upon startup that changing a file's location disables script functionality. After a discussion with the client about the issues of file relocation, it was clear that she understood the issue enough to be mindful of it without the reminder and this requirement was discarded. This functionality instead was handled by implementing a Config.txt file in which the user can specify the path names to new file locations without altering the code. Another secondary nonfunctional requirement requires the tool to run to completion within five minutes. While this was not a high priority or critical requirement, the output successfully assesses data within five minutes. This may change as the rate of new data collected increases, but upon completion of this project, this requirement was met.

The scripts produced in this project also had to produce a summary of the number of data issues found. Originally, planned to be a PDF, this requirement was instead met from within the script with a printout report given to the user upon completion.

Finally, all scripts must clean up unnecessary files and fields. This helps avoid cluttering up incoming data with extra fields and filling storage space. This final requirement was met on all four scripts. 
Table 1. Project requirements

\begin{tabular}{|c|c|c|c|}
\hline Script & Requirement Description & Type & Priority \\
\hline $\begin{array}{l}\text { Cluster Sightings } \\
\text { Identifier }\end{array}$ & $\begin{array}{l}\text { Tool shall flag any MMOPs that } \\
\text { have similar time stamps, the } \\
\text { same species, and fall within a } \\
\text { specified distance of each other. }\end{array}$ & Functional & Critical \\
\hline On-Land Identifier & $\begin{array}{l}\text { Tool shall flag any MMOPs that } \\
\text { fall within the borders of a land } \\
\text { feature. }\end{array}$ & Functional & Critical \\
\hline $\begin{array}{l}\text { Out-Of-Range } \\
\text { Identifier }\end{array}$ & $\begin{array}{l}\text { Tool shall flag any MMOPs that } \\
\text { fall outside a habitat range. }\end{array}$ & Functional & Critical \\
\hline Species List Creator & $\begin{array}{l}\text { Tool shall take latitude and } \\
\text { longitude inputs and produce a list } \\
\text { of species expected within a } \\
\text { specified distance. }\end{array}$ & Functional & Critical \\
\hline All 3 Identifier Scripts & $\begin{array}{l}\text { The tool shall remind user on } \\
\text { startup that if file structures have } \\
\text { changed the tool will not work. }\end{array}$ & Functional & Medium \\
\hline All 3 Identifier Scripts & $\begin{array}{l}\text { Tool shall produce a summary of } \\
\text { flagged points for the user upon } \\
\text { completion. }\end{array}$ & Functional & Low \\
\hline All 4 Scripts & $\begin{array}{l}\text { Tool shall clean up unnecessary } \\
\text { files and fields upon completion. }\end{array}$ & Functional & Low \\
\hline All 4 Scripts & $\begin{array}{l}\text { Tool shall complete running } \\
\text { within } 5 \text { minutes. }\end{array}$ & $\begin{array}{l}\text { Non- } \\
\text { Functional }\end{array}$ & Medium \\
\hline
\end{tabular}

\subsection{System Design}

After closely examining the requirements set, the next step in the project was to create a system design. This project required an ArcGIS for Desktop license and installation of an integrated development environment (IDE) that can handle Python code. ArcMap and ArcCatalog were required for producing the shapefiles and handling sample data. These products would also be key in checking the results of the scripts to be sure they were handled correctly. The IDE chosen for this project was PyScripter, a program that easily handles Python code and offers a color-coded interface with multiple debugging capabilities such as variable windows, an interpreter window, and break points. 
The individual components on the computer included a File Geodatabase containing sample observation points, habitat range polygons, and worldwide shoreline feature classes. In order to create the range polygons, a worldwide ocean feature class was also required for the production phase, though it could be discarded upon completion of the ranges. This File Geodatabase was contained within the same folder with the Data Checker scripts and the Species List Creator script.

\subsection{Project Plan}

The original plan for this project included four major stages: the project preparation, range feature class creations, script creation, and project completion. These steps were scheduled in a Gantt diagram (Figure 3-1).

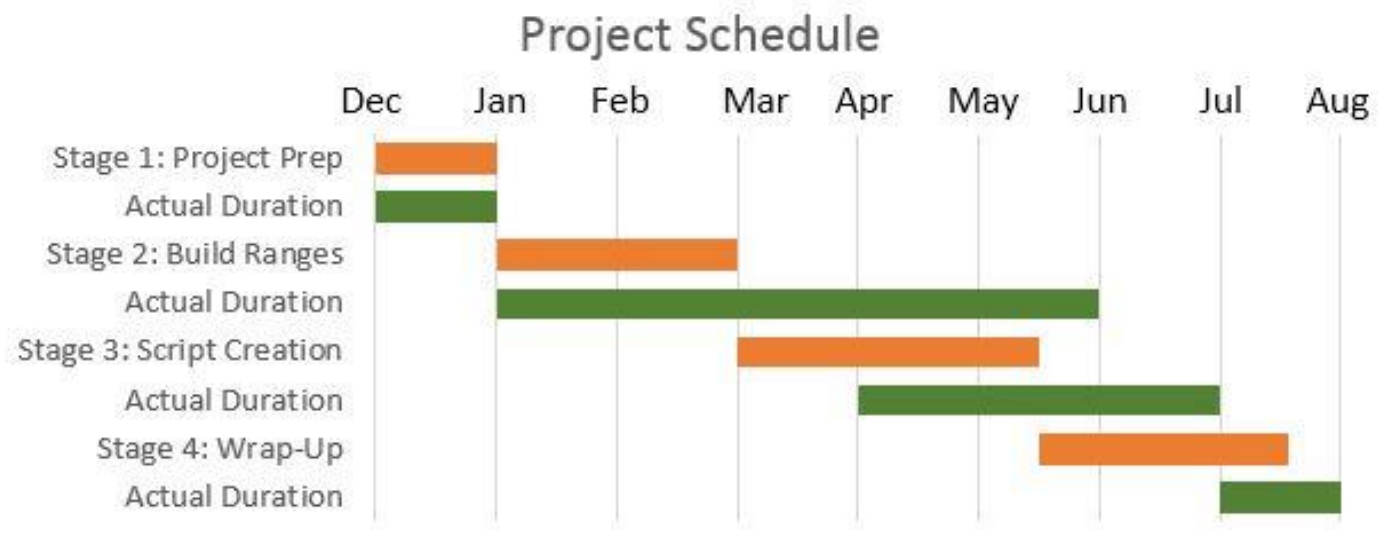

\section{Figure 3-1 The project schedule}

The first stage involved research and data collection and was successfully completed within the first month as intended. Data was quickly collected and prepared for use.

The second stage, creating the range shapefiles, took several months longer than expected, as the ranges were more difficult to create than expected. Each range had to be created manually, as all attempts to contact the authors of the ranges displayed online were unsuccessful. The project proceeded once all ranges were created for current Whale mAPP species and additional ranges were created during the following months during the next phase to complete a comprehensive list of species worldwide.

The third stage, creating the scripts, also took half a month longer than expected. Earlier script versions worked but needed to be adjusted in several ways to continue to comply with the original requirements. These final scripts were of higher quality and caught data issues with better precision than prior versions. All scripts work as intended and have been confirmed to catch all errors created in synthetic data and in the sample data.

The fourth script created in this stage was the Species List Creator. It assists the Whale mAPP programmer in populating a species list based on the range files for each new user rather than requesting the user to provide a species list.

Though the final stage occurred later than expected, it took less time to hand off the project than originally estimated. Instructions were handed off to the client along with the 
data and scripts from the project. The client has instructions on how to install the data and edit the Config.txt file to specify the new file locations.

\subsection{Summary}

At the start of this project, the goals appeared to be simple and straightforward. As the project continued, however, it became clear that initial anticipated scheduling plans were overly optimistic and the Python coding was more time consuming than originally thought. As the project evolved, overestimations emerged which balanced the underestimations. All critical requirements were met by the final script deliverables of this project, and the other requirements listed were either met similarly by the scripts or alternatively through communications with the client. The System Design planned for this project also met all needs expected throughout this project. Aside from the Range Updater tool, which was replaced with the Species List Creator, all original plans for this project progressed successfully to completion. 


\section{Chapter 4 - Database Design}

This chapter will explain the finer details of the project design. It will first examine the project's conceptual data model. It will then discuss the logical data model. Data sources will be discussed, as will the collection method. Data cleaning and loading will also be briefly touched upon. The chapter will conclude with a summary of key information from the chapter.

\subsection{Conceptual Data Model}

The conceptual model produced for this project built upon earlier Whale mAPP work (King, 2012) and displayed the relationships between the prior work and the new quality control work done in this project. Figure 4-1 shows the model with prior work displayed in blue and new work from this project displayed in green. Four key components existed prior to this project. The public users have spatial information of where their journey begins and where they are during their trip. The public users make observations that have species information and date and time information. These observations include the spatial information delivered by the public user during the trip and the identification of marine mammals. More than one marine mammal can be identified with each observation since the user may be observing a group of individuals. There may be many observations for each marine mammal individual or group when multiple users observe the same individual or group. There may be more than one observation per person, but there can be only one user per observation. Observations are made by public users with the help of the Whale mAPP application.

This project introduced several new classes to the conceptual model. Previously, users had to specify a species list to be programmed into the Whale mAPP. This process is much easier with the script made in this project. The project also introduced the concept of processed observations, which identifies potential data issues via the data checker scripts created in this project. 


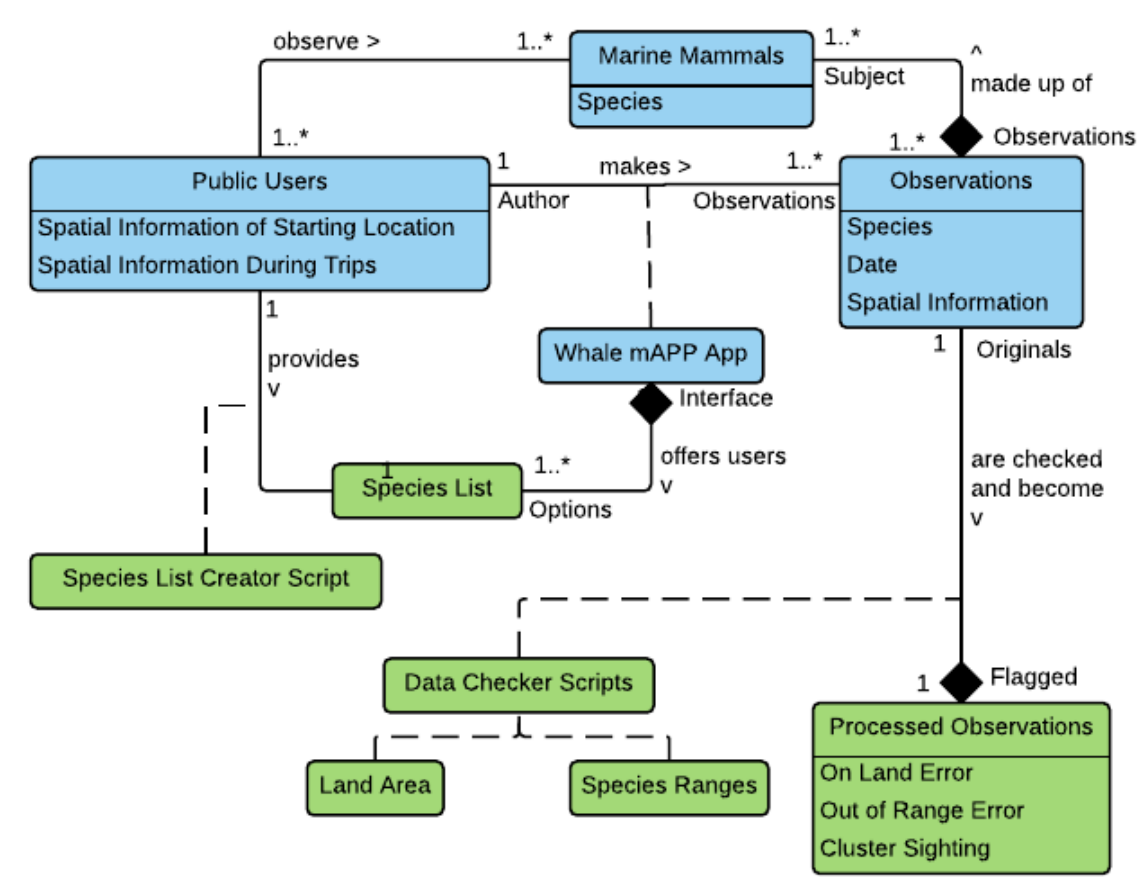

Figure 4-1 The conceptual data model: prior work (blue) and new work (green)

\subsection{Logical Data Model}

Once the conceptual data model was completed, the next step in the project was to think about the process in a logical model (Figure 4-2). This logical model takes a closer look at the GIS aspects of the project by examining the attributes in each feature class. This model also summarizes Whale mAPP conceptual themes within one Whale mAPP feature class and better displays the file structure applied by this project.

The highest structure shown on the logical model is the geodatabase. Within this geodatabase, there are three feature classes: the land feature class, the ranges feature class, and the Whale mAPP feature class. The land feature class contains a single feature representing all land around the world. The range feature class contains a feature representing expected habitat range for each species of marine mammals. The Whale mAPP feature class contains all observation points from the Whale mAPP, from both points processed by the scripts and from new incoming data.

The attributes for these three feature classes are also identified in this logical model. The land feature class had no attributes so was left blank in the logical model. It was only important to the scripts spatially and used to check overlap characteristics. The ranges feature class was created as a part of this project. While it was based on a pre-existing oceans feature class, several attributes were added. The Genus, Species, CommonName, and AnimalCategory attributes identify what species the range were created to match. The Source and SourceDate fields identify the source of the range information and when it was published. The UpdateDate and UpdateName attributes identify who last updated the range and on what date it was updated. All of these fields are text fields. 
The Whale mAPP feature class was a pre-existing file extracted from the Whale mAPP dataset. The file features various observation information such as the number of calves seen, the weather, the animal's behavior, or a group count. These attributes were not important to this project and were not included in the logical model. Additional original attributes such as the SHAPE@XY geometry field, the Date field, and the SpeciesType text field contain information vital to the project, so they were included in the logical model. In addition, once the scripts have run at least one round of checks on this file, the file gains several new attributes: an OnLandError field, a RangeError field, a DistanceFromRange field, and a SightingCluster field. These four fields receive the results of the data check scripts and describe whether an observation received any issue flags or whether the observation was checked and passed each script. All of the attributes vital to the project were included in this model.

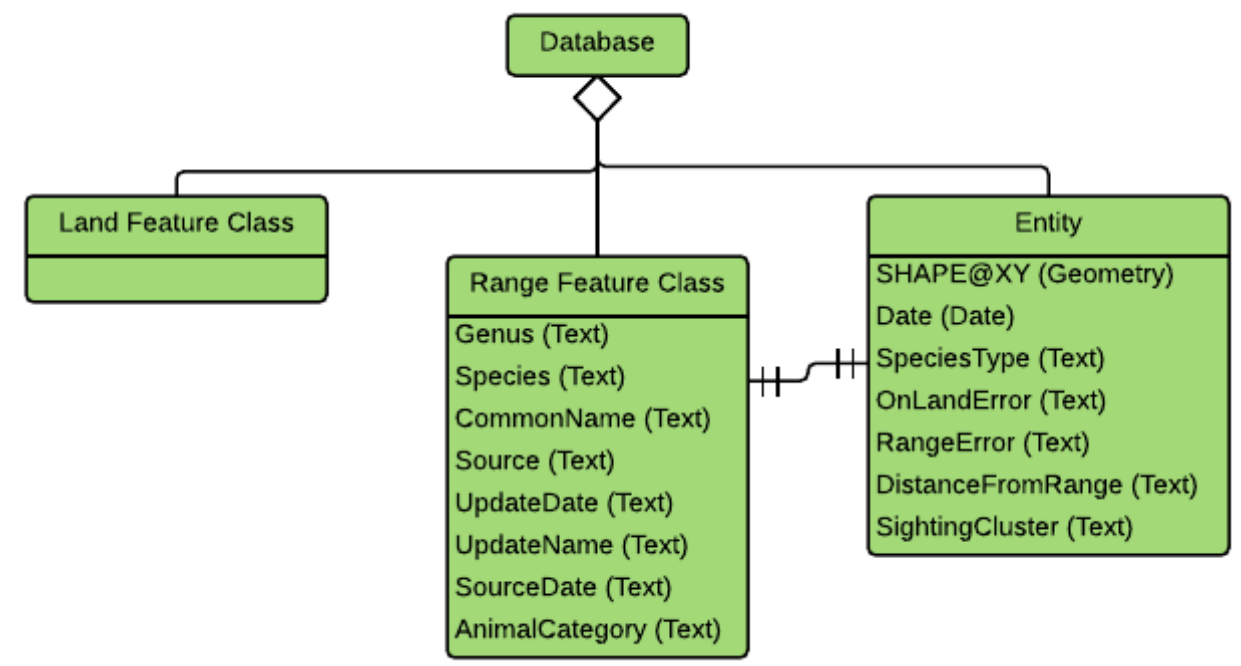

\section{Figure 4-2 The logical data model}

\subsection{Data Sources}

The three major feature classes used in this project came from several sources. A 10meter accuracy ocean shapefile was downloaded from the Natural Earth Data website (2013). Collecting the ocean shapefile was a simple click-to-download process. This dataset was then used to erase the ocean polygon from a worldwide polygon to create the land shapefile that was used for on-land testing. The ocean shapefile was listed as having a $10 \mathrm{~m}$ accuracy to true shoreline location and was derived from the CIA World Data Bank of 1977.

The ocean shapefile was also used as a basis for creating the ranges for each species. Multiple books and online sources were discussed in detail with the client and she recommended several sources for marine mammal range information. The two sources used in this project included the NOAA Fisheries Marine Mammals information page (NOAA, 2015). This page contains range information for 19 dolphins, three porpoises, 30 whales, two sea lions, and 15 seal species. All species of interest not available on this 
website were examined in Whales, Dolphins, and Other Marine Mammals of the World Princeton Field Guide (Shirihai \& Jarrett, 2006).

The sample Whale mAPP dataset was provided by the client. The data included all points to date that had been collected from the Whale mAPP. This provided an opportunity to test the scripts on real data. This dataset was easily received by downloading the dataset from a file share link. A secondary dataset was made synthetically to use during the testing phase. 160 points were extracted from the sample Whale mAPP data and moved to various spots that would or would not be expected to trigger an error script. There were 10 points to each potential error combination possible in the set of 160 points, including points that had no errors.

\subsection{Data Collection Methods}

Data collection in this project was minimal. The most significant effort was selecting range information for the ranges clipped manually from the 10-meter ocean shapefile. The NOAA Fisheries page was checked for range information for each species. If a species was not on the NOAA website, it was examined in the field guide. Each species page listed on this webpage also contained a link to a range PDF. The 10-m ocean was trimmed to match the range provided as closely as possible (Figure 4-3). The source PDF could not be georeferenced in ArcMap - which would have allowed higher accuracy or tracing abilities - due to unusual projections in the source files. This trimmed ocean feature was then moved into a new feature class containing all species ranges. Attributes were then entered to identify the species for each range, the source information, and notes about the most recent range edit. A discussion with the client about the species listed in the Whale mAPP ensured that the names of the species for each range would match the formatting of the observation species names provided by the Whale mAPP.

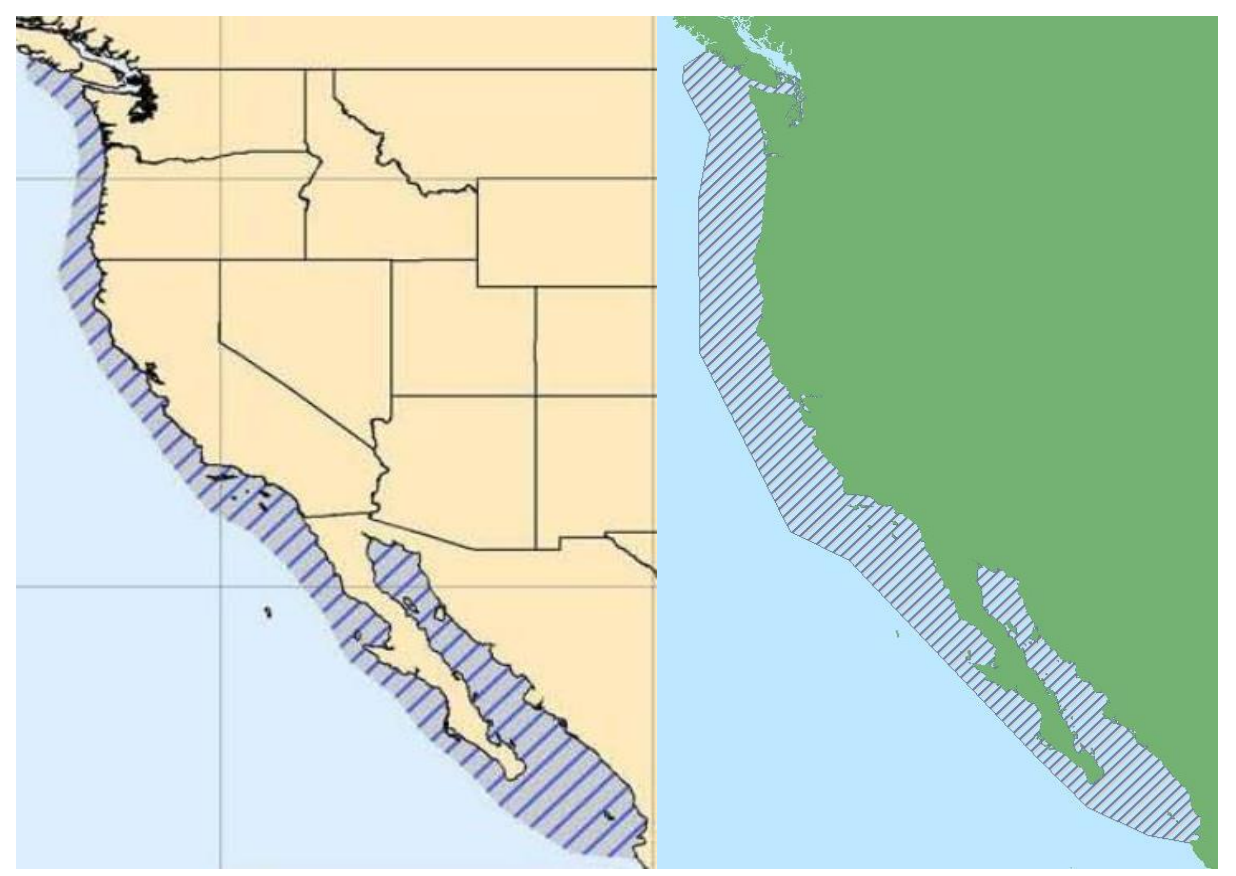

Figure 4-3 The California Sea Lion: source (left) versus feature created (right) 


\subsection{Data Scrubbing and Loading}

The Whale mAPP dataset collected for this project was clean upon being received. Table 2 shows a summary of the three data sources used for this project. Though marine mammal ranges change over time, the range information found both on the abovementioned webpage and field guide is assumed correct given the reputation of the sources. The files remain editable should end users choose to adjust the ranges due to changes in marine mammal behavior. While these files needed to be cut manually from a 10 -m oceans layer, no data scrubbing or loading occurred beyond this initial step.

Table 2. Data summary

\begin{tabular}{|c|c|c|c|c|}
\hline Data Name & Type & Method & Accuracy & Scale \\
\hline Ocean & Digital Shapefile & Downloaded & 10 -meter & Flexible \\
\hline $\begin{array}{l}\text { Ranges from } \\
\text { NOAA }\end{array}$ & Digital Static PDF & $\begin{array}{l}\text { Visual } \\
\text { Examination }\end{array}$ & Unspecified & $\begin{array}{l}1: 75,000,000 \\
\text { to } \\
1: 175,000,000\end{array}$ \\
\hline $\begin{array}{l}\text { Ranges from } \\
\text { Princeton Field } \\
\text { Guide }\end{array}$ & $\begin{array}{l}\text { Hard-Copy Book } \\
\text { Pages }\end{array}$ & $\begin{array}{l}\text { Visual } \\
\text { Examination }\end{array}$ & Unspecified & $\begin{array}{l}1: 50,000,000 \\
\text { to } \\
1: 500,000,000\end{array}$ \\
\hline
\end{tabular}

\subsection{Summary}

Both a conceptual model and a logical model are essential to approaching a problem in an efficient and successful manner. Initial data collection involved researching information about marine mammal habitats and fetching sample data from the Whale mAPP database. Data handling was minimal during the early stages of the project aside from manually creating the range information for each species. The dataset was handled more during the processing phase when it was edited and adjusted to clear out any changes made by the Python scripts until the scripts were completed. The majority of the work of this project will be discussed in the following chapter, which discusses the creation and testing of the scripts. 



\section{Chapter 5 - Script Development}

The implementation of this project was split into two sections. The first encompassed the most important part of the project: the data quality checks. The final section included the work done for the species list creator.

\subsection{Quality Check Scripts}

The quality check scripts were implemented through planning, debugging, and testing. While the original plan for this project included three separate scripts, the final version features only one script that runs all quality checks on incoming data in one run. Figure 5-2 shows a summary of the quality check script. The five stages include the startup, on land checker, out of range checker, cluster checker, and the wrap-up.

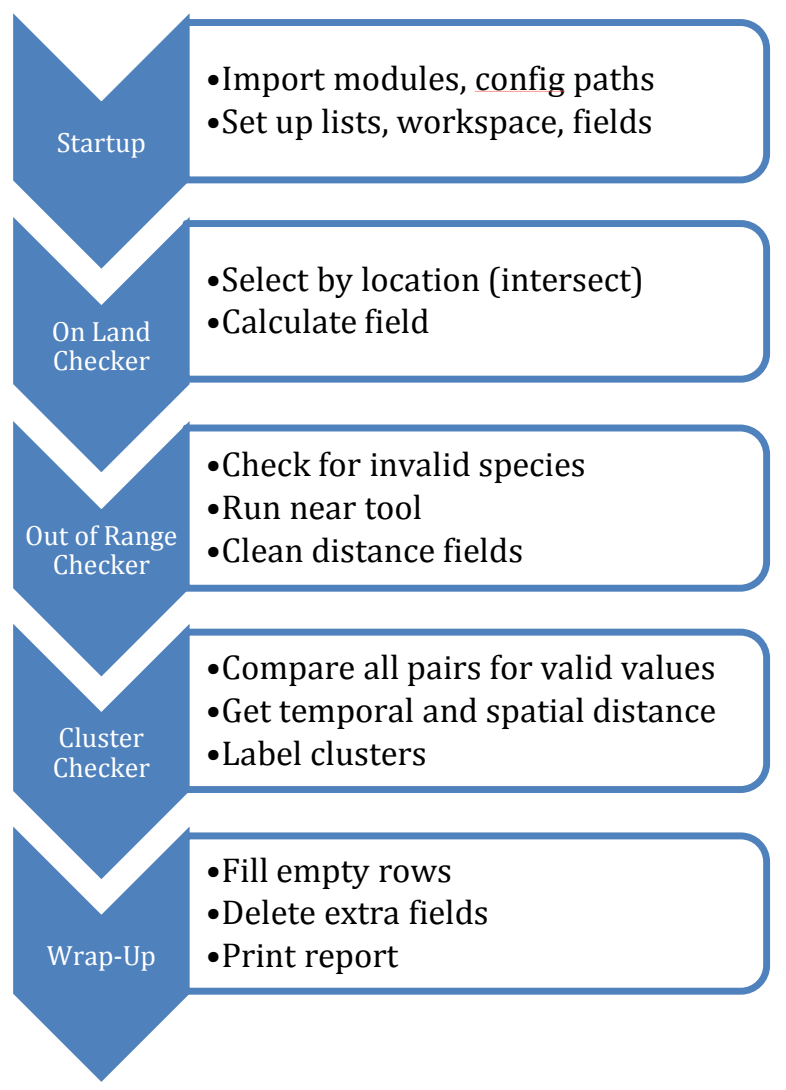

Figure 5-2 Summary of the quality checker script 


\subsubsection{Startup}

The startup step of the script performs all actions required for the rest of the script to run smoothly. First, necessary modules must be imported, including ArcPy, Time, DateTime, Math, CTypes, and OS. These libraries use specialized commands in a more efficient manner further down in the script. The startup also includes reading configurations set during installation of the script and related files. Python scripts require direct path names to the datasets to be used in the script. When installing the file, a Config.txt file must be edited to show the correct path files (Figure 5-3). Any information after the equal sign must be replaced with any new pathfile. The code then reads these files to collect the information needed to run commands.

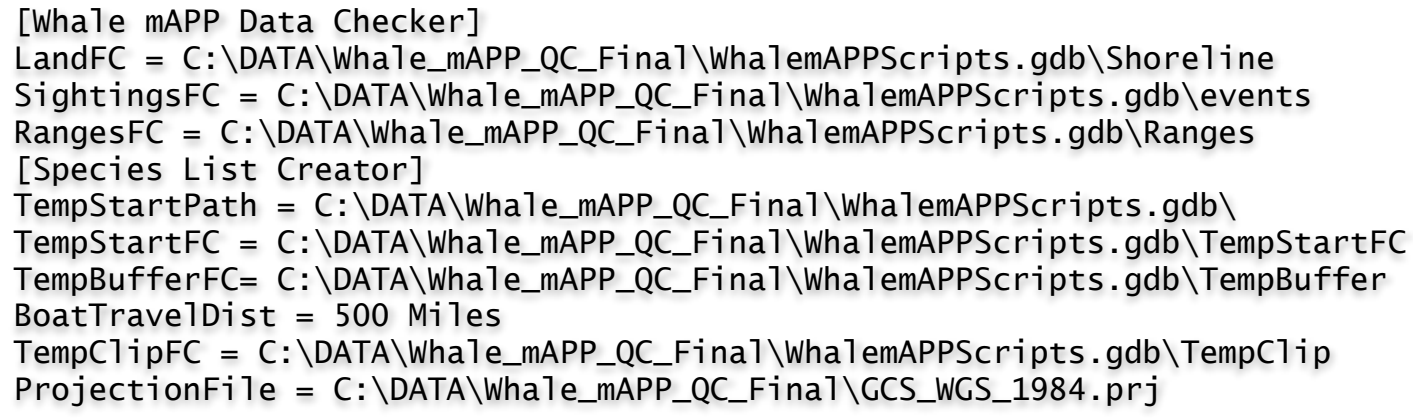

\section{Figure 5-3 The Config.txt file contents}

Finally, the set up phase creates required lists and fields in preparation for the quality checks. These fields include the results fields in the dataset. If the fields already exist, the script skips this step. 


\subsubsection{On Land Checker}

This section of the script checks the incoming Whale mAPP dataset for any points with on-land coordinates (Figure 5-4). In order to do this, the script uses a Select by Location command on the observations, represented by the SightingsFC variable, to select any points that overlap landmasses, represented by the LandFC variable, by using the selection type "Intersect." The Field Calculator command from the ArcPy library is then used to populate all selected points with "Sighting Is On Land" in the OnLandError field.

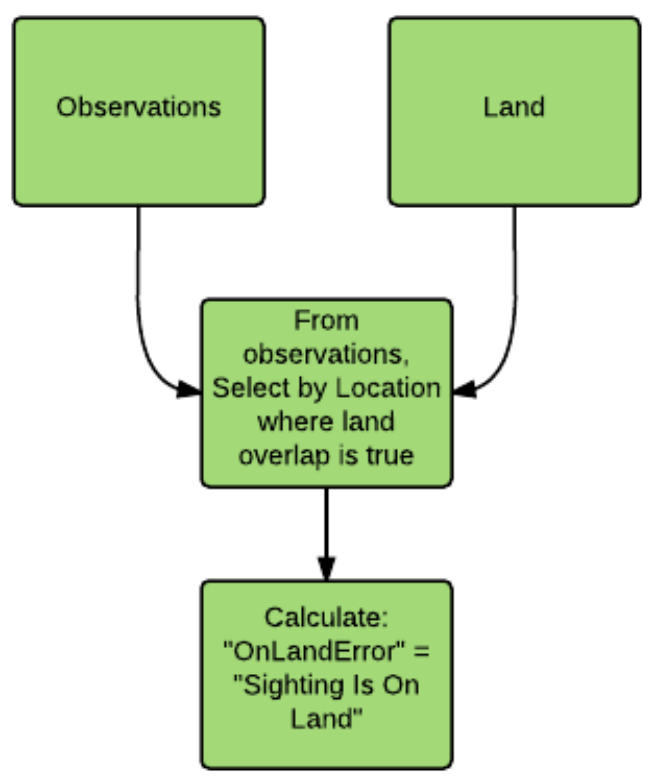

\section{Figure 5-4 The On Land Checker workflow}

\subsubsection{Out of Range Checker}

The out of range checker uses a combination of lists and selection rules to test all observations of each species in the Whale mAPP dataset (Figure 5-5). When checking the observation dataset against the ranges available, a list is created for species names that do not match a range (invalidSpecies). These observations are labeled as "No Range Match" in the RangeError field. A list is then created of all species found in the observation dataset that do match a range (observationSpecies). Once the observationSpecies list is created, selections are made from both the observations and the ranges for each of the species.

These two selections are processed through the Near analysis command in the ArcPy module that checks the distance between two inputs. If observations fall within the species range, their distance is set to zero. If an observation falls outside a range, it is given the distance in meters to the closest edge of the range. Finally, because the Near tool will calculate all other observations as negative ones if they do not match the current species being checked, all the correct values for each species are moved to a new field until all species have been checked. The final output includes negative one for all species 
listed as "No Range Match" from the first part of this step, zeros for all species found within range, and values greater than zero for any observation out of range. All observations with values greater than zero are run through a Field Calculator to list the RangeError as "Sighting Is Out Of Range."

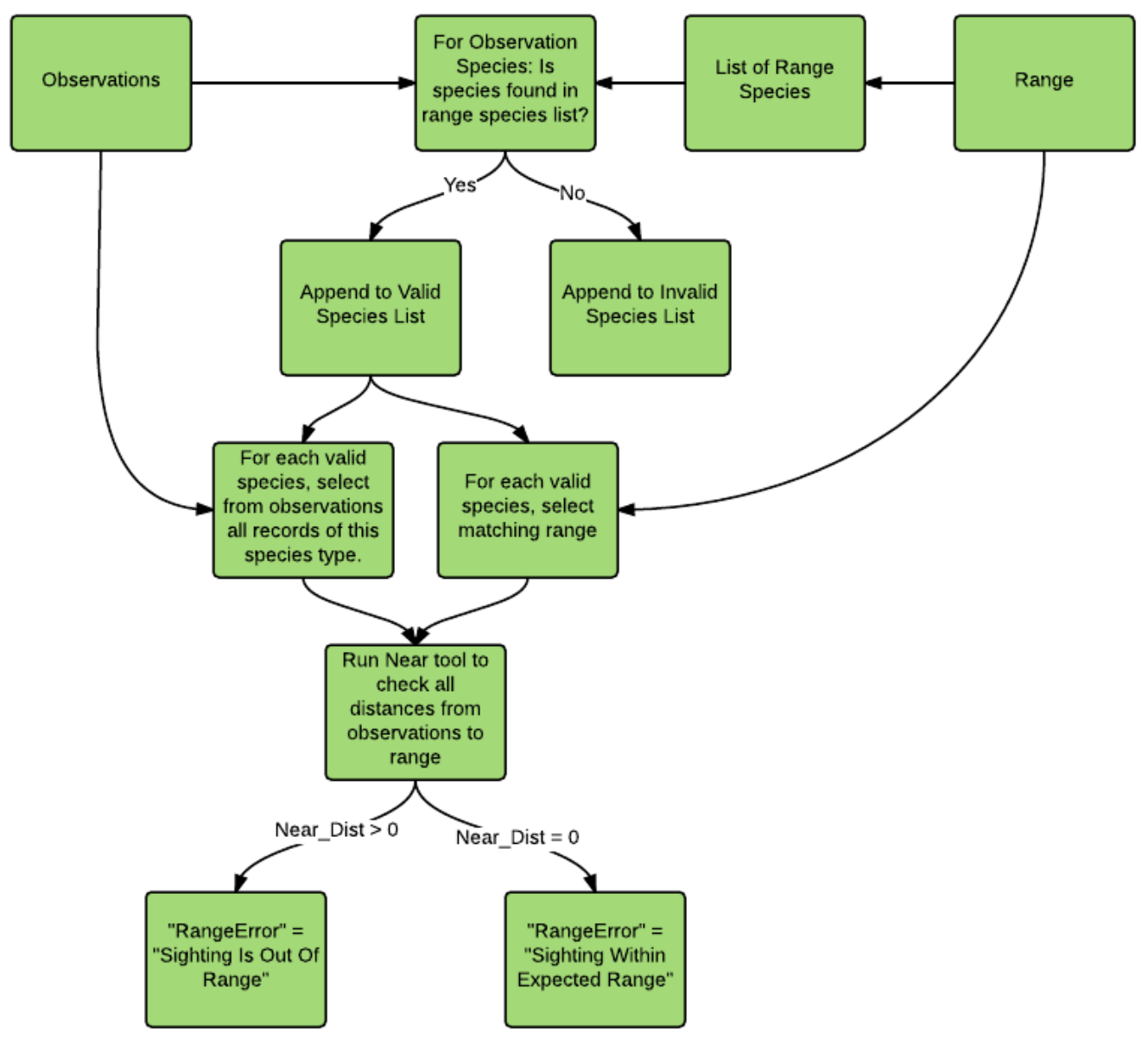

\section{Figure 5-5 Out of Range Checker workflow}

\subsubsection{Cluster Checker}

The cluster check part of the script takes the SightingsFC observations and compares each observation against all others to check if any of the data are similar enough to skew the database (Figure 5-6). First, the script sets up two cursors since two observations need to be compared. While the first cursor gathers field values for the first sighting, the second cursor loops through all other sightings and gathers information about each sighting as it passes. Values are then examined by comparing the time and date information, the sighting ID, the species name, and the latitude and longitude for each observation. 
The script lowers the runtime duration by first ruling out unnecessary checks. If the unique observation IDs for each point are the same, the second cursor moves on to the next point to avoid labelling every observation as a cluster with itself. If the first cursor's observation has no time and date value, it labels the observation as having an "Invalid Date/Time" in the SightingCluster field, and moves on to the next observation. If the second cursor receives no time and date value, the second point is skipped. If the species names of the two observations are not the same, then the points are skipped, as well. By avoiding the extra time it takes to check certain points, the script runs faster while still giving accurate results.

The script then uses the Time and DateTime modules to manipulate each point's date and time information into a time tuple. Time tuples can be manipulated to find a difference in time by subtracting one tuple from another and getting the absolute value of the result. This value is stored in the TimeDifference variable for later.

The script also checks spatial distance by using the Pythagorean Theorem and the Math module. The difference in latitude and difference in longitude are added together and stored in the addedXYDifferences variable. The Pythagorean Theorem states that square rooting this value provides the distance between the two points opposite the right triangle formed between the latitude and longitude lines. Because taking the square root of this value takes more time than a quick comparison, this value is left squared and compared in the next step as-is.

The script finally uses these two variables - the TimeDifference and the addedXYDifferences - to determine if two points are similar enough to consider them a cluster. The points must have occurred within one minute of each other and within 500 feet of each other. In this case, a comparison is made against (500 meters) $)^{2}$ or 250,000 meters $^{2}$. If TimeDifference is smaller than one minute and addedXYDifferences is less than 250,000 meters $^{2}$, the points are clustered. Points are spatially clustered if they are within 500 meters of each other. In order to speed up the script's calculations, instead of asking the script to square root the addedXYDifferences, the 500-meter distance is squared to 250,000 before being compared. When points are considered a cluster, the SightingCluster is calculated as "Cluster Found." 


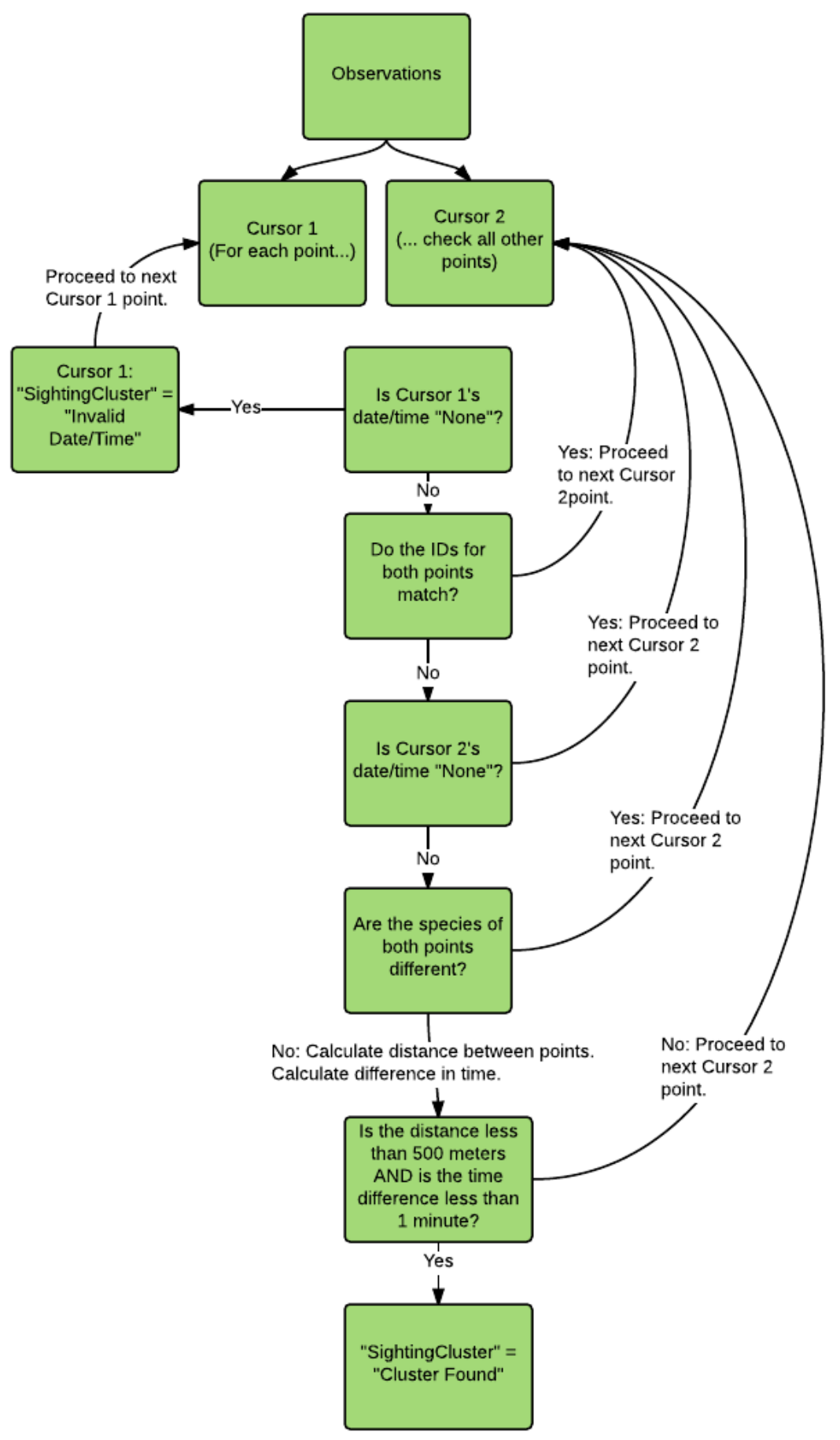

Figure 5-6 Cluster Checker workflow 


\subsubsection{Wrap-Up}

The last section of the code includes all wrap-up tasks. The script first examines the dataset, populates empty error fields, and edits the wording of the outputs in the proper fields to ensure that the end user will understand what these outputs signify. All observations without a matching species range are labeled as "No Matching Range Found" in this step. Observations with distance equal to zero are marked "Sighting Within Expected Range." If a distance above zero is given, the field is recalculated to include "Meters From Range" for easier reading. If an observation does not trigger a flag in any of the other error fields, the error field is labeled as "Checked."

Extra fields are also deleted in this step, including the two output fields from the Near Tool (Near_DIST and Near_FID) and the temporary distance field that stored the proper distance from the range edge. This helps tidy the processed Whale mAPP file for easier use after the script is complete.

The final task of the script is to print a summary of errors found to the user after completion. This summary includes the number of observations that were checked, the number of flags triggered found for each type of data issue, and a percentage of data that includes one or more flags. Printing this summary offers the user feedback about the reliability of the dataset once it has been processed.

\subsection{Species List Creator}

The Species List Creator script was made as a tool for use when programming new users' data into the Whale mAPP application. Prior to this script, a new user had to research expected species in the study area and send a species list to be programmed into the application. This script requires only a start location and uses the range files made for this project to populate a species list. The start location can be, for example, the harbor from which a whale watch will depart. Because the Whale mAPP requires JSON-format programming inputs, the output of the script produces a JSON-format list of species. This script has three steps: the startup, the species list creation, and the wrap-up (Figure 5-7).

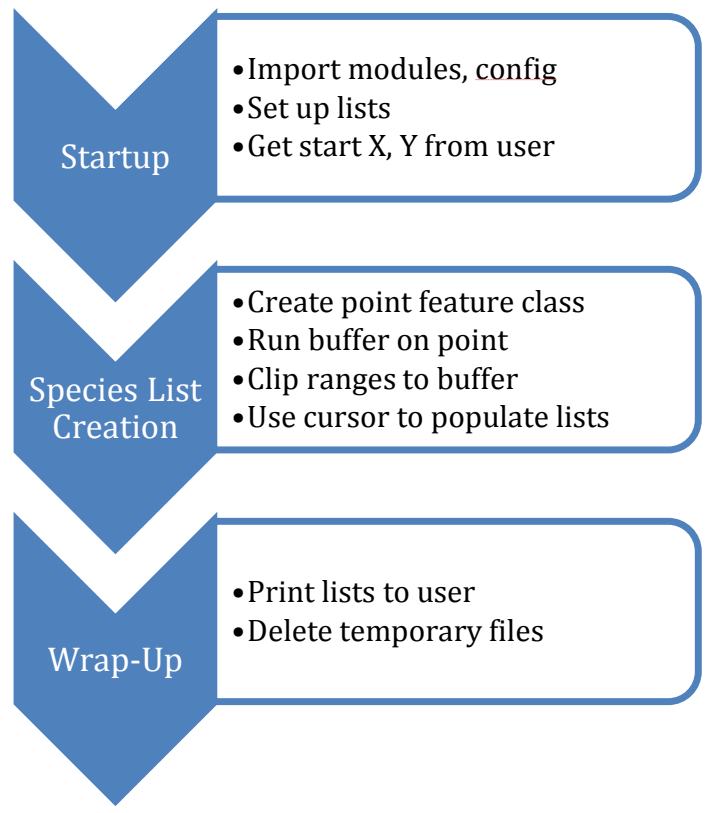




\section{Figure 5-7 Summary of the species list creator script}

\subsubsection{Startup}

The code starts by importing the ArcPy module and Config.txt file showing the path names to input files and temporary and final outputs. Many of the steps contained in this script require input and output file locations. When a file already exists, the code produces an error during a run through and quits the script. Setting up input and output file locations allows later deletion of these intermediate files, thereby avoiding this error. The initial step also sets up required lists that are used later in the script. Introducing these lists at the startup phase helps keep the code more organized.

The startup also prompts the user for the latitude and longitude of the new Whale mAPP's starting location. This would typically indicate a harbor, dock, or similar edgeof-water location where a whale watch would begin. If the new user is unable to provide this location, a quick search using Google Maps or other similar X-Y search tool can be used once the user provides the start location by name.

\subsubsection{Species List Creation}

The second section of the script is the main part, where the majority of the work takes place. In four steps, a new species list can be produced from the input X-Y coordinates. First, the script creates a new point feature class with a single point representing the starting location provided by the new user. The Buffer analysis command is then run on this feature class, creating a circle with a radius equal to the distance specified in the Config.txt file. The initial distance is 500 miles and represents a search area for any species that may pass into the boat's potential travel distance. This buffer is then saved as a temporary polygon feature class. The script then uses Clip analysis to extract all ranges that are contained within that buffer circle into a new polygon feature class. The species included here are the species a user can expect to see during his or her trip. Finally, a cursor is used to run through all ranges included in this final feature class, reading the species names into the lists created at the beginning of the script.

\subsubsection{Wrap-Up}

The final section of the code includes two steps in which temporary files are deleted and the lists produced are read to the script user. The script deletes the temporary point file representing the boat's starting location, the buffer circle representing the boat's potential location during a trip, and the feature class containing the ranges extracted using the buffer. Removing these three files prevents storage issues and "File already exists" errors. The final step in this script is to print the completed species list to the user. An optimal output format was determined so that the results can be copied directly into the programming for the Whale mAPP for easy use via JSON code. The output is separated 
by type of marine mammal so that all whales, seals and sea lions, dolphins and porpoises, and other marine mammal species such as otters and polar bears are kept together.

Through this process, the Species List Creator script optimizes new user additions by automating the process of species list creations by location rather than by new user input. Because many of the future users of the Whale mAPP may not be familiar with the area, they may not know what species to expect. This allows a more accurate list to be produced for the users simply by being provided a start location.

\subsection{Summary}

Programming the scripts for this project encompassed the majority of the work and produced the most important outputs of this project. The quality check scripts had several bugs early on within each component and it took a large amount of testing and debugging to complete them successfully. The species list creator script was less problematic but relied on the full completion of all range features to be truly useful to the end users. The scripts were successfully wrapped up into two separate software that can be run through PyScripter, and they are now ready to be utilized. 



\section{Chapter 6 - Results and Analysis}

Prior to this project there was no method for performing bulk evaluations of incoming data from the Whale mAPP. The Whale mAPP data stands at 700 points but will increase rapidly with more beta users and upon release to the public. Checking each point manually would be both unreliable and unrealistic. This project allowed for quality assessment of the incoming crowd-sourced dataset. When a dataset has been analyzed for data quality, it can be used with more confidence by researchers.

\subsection{Error Count Analysis}

The sample dataset used to test the Whale mAPP's quality included points collected between October 26, 2013 and November 17, 2014. During this period 720 points were collected. Forty-three percent of the observations contained one or more flags (Table 3). This was a relatively high percentage of data with errors. Almost $35 \%$ of the dataset are observations with species names that do not match the current ranges available. This issue will be diminished over time as standardization occurs between the range and observation species names. The names in the Whale mAPP have been updated to match the ranges names, though this change occurred after the last observation included in this project. Since the species lists will be populated from the species names in the range file using the Species List Creator script, this will also help standardize the application. Users will still have the option to submit observations with unique names by selecting "Other" on the application, but this will become less frequent.

Observations submitted with no date and time information only occurred in about $5 \%$ of the current data. If both of these errors are disregarded from the sample, the percentage of data with errors drops from $43 \%$ to $13 \%$. If that projection holds true, then for 720 points collected, 626 points would be usable with a high level of confidence. While the Whale mAPP is still in Beta release with a select number of users, once it is released to the public the number of points collected per year will grow significantly.

\section{Table 3. Summary of results}

\begin{tabular}{|l|l|}
\hline & \\
Dataset In Its Current State & Dataset Without Name or Date Issues \\
\hline Points on land: 27 & Points on land: 27 \\
Points out of range: 20 & Points out of range: 20 \\
Clusters found: 47 & Clusters found: 47 \\
No matching ranges: 249 & No matehing ranges: 249 \\
No date/time information: 37 & Nodate/time information: 37 \\
Total percentage of flagged data: $\mathbf{4 3 \%}$ & Total percentage of flagged data: $\mathbf{1 3 \%}$ \\
\hline
\end{tabular}

One must also consider that while clusters may skew studies based around population densities, clusters are in fact not an error but rather too much good data. If cluster concerns are removed from the evaluation, this drops the percentage of flagged data from $13 \%$ to $7 \%$, with 27 points on land and 20 points out of range. Of these 27 
points on land, 10 points had no range match, and 17 were labeled out of range. Of the 20 out of range points, 3 were out of range at sea while the 17 remaining points were already encompassed by the points on land values.

In this fashion, these quality control scripts help an end user evaluate the potential issues with the data prior to use in a study. Better data promotes better science and compared to the prior Whale mAPP data versions a user now has a better knowledge of data that was submitted with improper spatial information. A user can also identify points that may have been identified incorrectly, or perhaps may be of interest in studies that indicate species range changes as species may start to appear farther north than usual with changing water conditions. A user also can produce a more accurate estimation of population densities by checking the cluster flags. All of these factors were unidentifiable prior to this project.

As the Whale mAPP is released to the public, the number of users will grow as friends share the application, families get involved, researchers pick up the tool, and tourists start exploring the application to record their adventures. With more users, the number of observations will also grow exponentially. Data users may quickly have become overwhelmed with the number of points they receive, uncertain on the reliability of the data. Many people, faced with uncertain data quality, may choose to disregard the dataset entirely rather than take chances that data may be incorrect. With this project, a solid percentage can be produced and offered to end users. Data requests can be handled by querying error types out of the dataset, thus catering to the needs of the end user, an ability that many crowd-sourced datasets are unable to offer.

\subsection{Potential Inaccuracies}

Several inaccuracies were identified during this project. The first inaccuracy was associated with the imprecise shorelines depicted in the output data. A higher accuracy shoreline leads to greater accuracy of error flagging. A high-accuracy shoreline dataset was found on the NOAA National Center for Environmental Information website (2015). This dataset was the inverse of what the project required for range files - a worldwide ocean layer. During multiple attempts to create an ocean layer from a file with high resolution, the computer froze and ArcMap crashed, unable to handle the request.

Instead, an approach was taken to find an ocean layer first. A 10-meter accuracy ocean shapefile was found on the Natural Earth Data website. Although the project could have used the land shapefile mentioned earlier in combination with this new ocean shapefile to create the ranges, the edges between the two datasets did not match, causing some points to be labeled as in range but located on land. To avoid this issue, a worldwide land feature class was made as an inverse of this ocean layer. The lower resolution allowed the creation of the land features without freezing ArcMap.

The range files also had accuracy issues due to the nature of manual creation. Unusual map projection properties made it difficult to geo-reference these images into ArcMap without severe distortion of the image. Attempts were made to contact the authors of the sources in the hope of collecting the digital shapefiles for this project, but they did not reply. Many of the hard-copy source maps were also very small, which caused a loss of detail about range boundaries. Without receiving the data, the best possible job was done through visual inspection and replication. 


\subsection{Problematic Assumptions}

These quality checks for the incoming Whale mAPP dataset made several assumptions that may be problematic. The on land checker portion of the script uses a strict true or false response, which assumes that all marine mammals are found in water, and if they are found on land, the observation must be wrong. There are, however, exceptions for certain species. Seals and sea lions can be seen basking in the sun on shore. If a user on shore submits observations about these mammals, the points may be inappropriately flagged by the script as incorrect. These points also may cause issues as the shorelines collected have a 10-meter accuracy. Points close to land may have in fact been located to an on-land location, but with the 10-meter accuracy, may have registered as in water. These points would not be flagged for review. This could for example result in a humpback whale showing two meters off shore that was in fact mis-located. Users may also stand on land and enter observations about marine mammals spotted in the water. For example, whale spouts can be seen for great distances. Users may make observations of these whale sightings. The script would catch these points as located on land, while a marine mammal was legitimately seen off shore. This issue, however, is a problem with incorrect application use rather than a data error and may be addressed through in instructions to the user. The script is also intended for worldwide use, which would include polar bears, which can be properly observed on land.

The script assumes that any point out of range is an issue that needs to be flagged. The problem with this assumption is that as water conditions change or external forces occur, marine mammals will shift ranges. A particularly warm winter may allow some species to travel farther north than usual. Abundant food sources may encourage a species to gather outside of their normal area. New or intensified human activity may drive animals outside of their normal location. These points may represent correctly identified species simply out of their accepted range, but the script would treat these as problematic data.

Finally, when checking for clusters, several properties were defined by the client as the rules for a cluster. Two points must have the same species name, occur within a minute of each other, and have locations less than 500 meters from each other. There may be more clusters in the data that were not picked up from this flag that were, for example, 61 seconds apart, 501 meters away, or had the same species but one was typed in by the public user through the "Other" field and was capitalized differently. For example, one observer may have chosen the drop-down "Humpback whale" while another observer may have typed "Humpback Whale", which the script would not match. These points would not be identified as clusters. Similarly, points that occurred close together may not necessarily be clusters. Though two different observations have similar information, the users may simply have entered similar information at a similar time out of coincidence. For example, two whales recorded breaching off different ends of a boat may be labeled as clusters of the same sighting when the observations were about two different whales.

While all of these assumptions can put the dataset in danger of showing false positives, the data remain unchanged except for the additional error fields. The end users of the database may choose whether to use these points or discard them.

Ultimately, the decision about whether a point is correct or incorrect lies with the user; 
the scripts simply attempt to inform the user of potential errors so that the users are better informed about potential problems in the dataset. 


\section{Chapter 7 - Conclusions and Future Work}

This project completed what it set out to accomplish. The project began with a request for assistance in evaluating and handling crowd sourced data. Python coding was utilized to create a script to assist with evaluating the quality of incoming data from the Whale mAPP. This script checks observations against landmasses, habitat ranges, and other observations in the dataset. One facet of this project was meant to identify issues in connectivity where observations with land-based coordinates were submitted. The script flags points that are outside a species' typical habitat range, which may indicate an incorrectly identified species. The script then checks properties such as the identified species, date and time, and location of observations to flag groups of observations with similar properties in an effort to prevent data skew. With the ability to flag potential data issues in incoming datasets, the Whale mAPP can now equip researchers with higher quality data. This allows crowd sourcing to remain a viable option in collecting data for future marine mammal studies.

The Species List Creator script supports the Whale mAPP in several ways. Prior to this project, the client had to rely on a new user's knowledge of potential species around their starting location in order to add the user to the Whale mAPP. This project implemented reliable marine mammal sources to predict and generate a species list for each new starting location. The species list creator requires only a starting latitude and longitude to produce a list of all species expected within a 500-mile radius of the starting location. This list is produced in the correct format for input directly into the application, saving time and avoiding the uncertainty involved in untrained users attempting to guess what species they may see. This also helps standardize the names that are then received in user observations. Users are less likely to manually type in the name of a species if options are already available in the application's species list. Species names that are typed manually into observation fields can cause issues with checking observations against expected ranges. Thus, using this technique to populate species lists from the available ranges helps decrease the number of potential errors, increasing the quality of the incoming data. The client received the completed files and all requirements set at the start of this project were fulfilled.

Several steps may be taken beyond the scope of this project to increase the usability and accuracy of these Python tools, including dynamic species list generation; photo identification technology; higher resolution data; and the automated adjustment of ranges.

The coding done in this project is intended for a desktop computer with ArcGIS and PyScripter installed. The species list creator outputs a paragraph of text with proper coding to allow copy-paste transfers into the programming of the Whale mAPP. The next step in automating the quality control of this project is to implement this code within the application itself. The functionality of mobile devices to fetch a latitude and longitude when a signal is available is what drives the spatial information collected for each observation. This information can be used upon starting a whale watch trip by fetching initial coordinates and running the script within the mobile device itself. However, this process may involve storing a large amount of range data on the device. Potential solutions for this issue may involve fetching species lists on the fly using geoprocessing tools on a server. If this technique can be utilized, each new user would automatically 
receive a new species list when starting a trip, which would be ideal given the number of contributors associated with crowd-sourced projects. This would severely cut down on the amount of work associated with taking on new users.

New identification techniques are being explored around the world to assist in identifying marine mammal species. Current techniques include keeping a photographic library of individual animals, often with unique names or identification numbers. When marine mammals are observed by researchers, photos are taken of sightings, which are then compared against all photos available in a library in an attempt to find the unique name or identification number of that individual. Modern technologies are now being utilized through extracting patterns on flukes and fins and matching these against digital archives, in a way similar to modern facial recognition software (Gope, Kehtarnavaz, Hillman, \& Würsig, 2005). This technology could be used by comparing contributor photographs against other digital mediums. Patterns in these photographs can then identify the individual or rule out certain species. While this direction would include less work in geographic information systems and more work in programming, biology, and mathematics, it has potential to both improve the quality of data and offer more motivation for public use.

The accuracy of the quality checks created in this project can also be improved by implementing data with a higher resolution. The range files were created through extraction from a shapefile with 10-meter accuracy for the Earth's shorelines. Higher accuracy would help in properly identifying sightings as on land. The accuracy of range checks may also be increased by implementing pre-made ranges created specifically for use in guidebooks and online information pages. Over time more accurate digital sources for ranges may become available which could replace the ranges created in this project.

Ranges change over time due to outside forces such as water temperature changes, pollution, human activity, and shifts in food availability. These changes can be tracked and studied by using concave hull tools to produce bounding polygons for the observations collected each year. Figure 7-1 shows how concave hull studies can be implemented to examine the different distributions from year to year. The red points could indicate, for example, all sightings of California Sea Lions in 2015, while the blue points could display all sightings of California Sea Lions in 2025. If a concave hull polygon is produced for both datasets, one could speculate that water conditions might have changed enough to allow the species a more northern range. Programming could be 
utilized to produce these results, giving researchers a new way to use the Whale mAPP data.

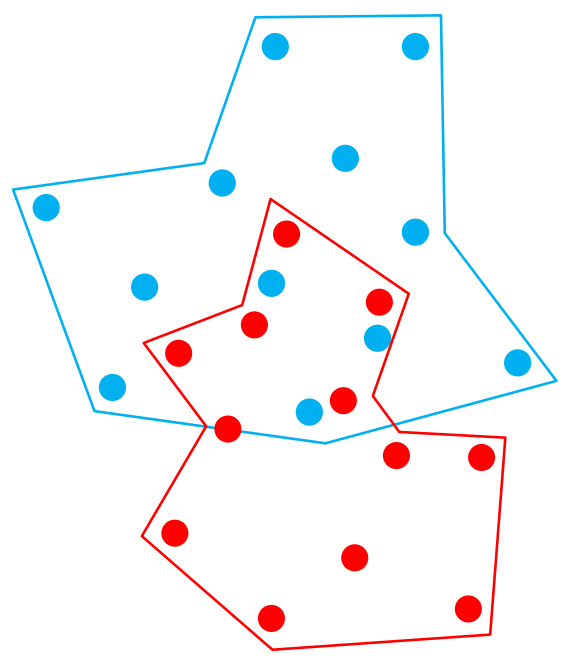

- Observations from 2015

Observations from 2025

\section{Figure 7-1 Hypothetical Concave Hull Analysis}

Many more ways in which the Whale mAPP application can grow may be discovered with its release to the public. As new users contribute to the database, more errors may be found. Users can submit requests for application changes. There may also be more ways to interact with the public and encourage participation. As the application grows and as more users submit observations, the dataset will become more valuable and researchers can then use Whale mAPP data to better understand the state of the world's marine mammals and oceans. 



\section{Works Cited}

1:10m Physical Vectors. (2013). Retrieved from www.naturalearthdata.com/downloads/10m-physical-vectors/

Carstensen Jr, L. W. (1998). GPS and GIS: Enhanced accuracy in map matching through effective filtering of autonomous GPS points. Cartography and Geographic Information Systems, 25(1), 51-62.

Chrisman, N. (2006). Development in the Treatment of Spatial Data Quality. In Devillers, R. \& Jeansoulin, R. (Eds.), Fundamentals of Spatial Data Quality (pp. 2130). London: ISTE Ltd.

Coleman, D. (2013). Potential Contributions and Challenges of VGI for Conventional Topographic Base-Mapping Programs. In D. Sui, S. Elwood, \& M. Goodchild (Eds.), Crowdsourcing Geographic Knowledge: Volunteered Geographic Information (VG) in Theory and Practice (pp. 245-263). London: Springer.

D’Antonio, F., Fogliaroni, P., \& Kauppinen, T. (2014). VGI edit history reveals data trustworthiness and user reputation.

Decker, D. (2001). GIS Data Sources. John Wiley \& Sons.

Goodchild, M. (2007). Citizens As Sensors: the World of Volunteered Geography. GeoJournal, 69(4), 211-221.

Gope, C., Kehtarnavaz, N., Hillman, G., \& Würsig, B. (2005). An affine invariant curve matching method for photo-identification of marine mammals. Pattern Recognition, 38(1), 125-132.

Gorter, U. (2002). Bottlenose Dolphin [website]. Retrieved from http://acsonline.org/factsheets/bottlenose-dolphin/.

Gorter, U. (2002). Franciscana [website]. Retrieved from http://acsonline.org/factsheets/franciscana/.

GSHHG - A Global Self-consistent, Hierarchical, High-resolution Geography Database. (2011). Retrieved from https://www.ngdc.noaa.gov/mgg/shorelines/gshhs.html

Haklay, M., Basiouka, S., Antoniou, V., \& Ather, A. (2010). How many volunteers does it take to map an area well? The validity of Linus' Law to volunteered geographic information. The Cartographic Journal, 47(4), 315-322.

Hijmans, R.J., Schreuder, M., De la Cruz, J., \& Guarino, L. (1999). Using GIS to check co-ordinates of genebank accessions. Genetic Resources and Crop Evolution, 46 (3), 291-296.

IUCN Redlist (2014) Spatial Data Download [website]. Retrieved from http://www.iucnredlist.org/technical-documents/spatial-data.

Kerski, J. \& Clark, J. (2012). The GIS Guide to Public Domain Data. Redlands, CA: Esri Press.

King, M. (2012). Managing Marine Mammal Observations Using a Volunteered Geographic Information Approach. (Master's thesis, University of Redlands).

Retrieved from http://inspire.redlands.edu/gis_gradproj/159/.

Koukoletsos, T. (2012). A Framework for Quality Evaluation of VGI linear datasets (Doctoral dissertation, UCL (University College London)).

Marine Mammals. (2015). Retrieved from www.nmfs.noaa.gov/pr/species/mammals/.

OpenStreetMap (2004). Retrieved from www.openstreetmap.org/. 
Perry, W. (1991). Quality Assurance for Information Systems: Methods, Tools, and Techniques. Boston, MA: QED Technical Publishing Group.

Stein, A., Shi, W., \& Bijker, W. (2009). Quality Aspects in Spatial Data Mining. Boca Raton, FL: CRC Press.

Steinberg, L. \& Steinberg, S. (2015). GIS Research Methods: Incorporating Spatial Perspectives. Redlands, CA: Esri Press.

United Nations. (1998). GIS Standards and Standardization: A Handbook by United Nations, Economic and Social Commission for Asia and the Pacific. United Nations.

Veregin, H. (1992). Data Quality Parameters. Geographic information systems, 1, 177189.

Vidal-Filho, J.N., Lisboa-Filho, J., de Souza, W.D., \& dos Santos, G.R. (2013).

Qualitative Analysis of Volunteered Geographic Information in a Spatially Enabled Society Project. Computational Science and Its Applications, 378-393.

Wikimapia. (2012). Retrieved from www.wikimapia.com/. 